\title{
Acidity/Reducibility Dual-Responsive Hollow Mesoporous Organosilica Nanoplatforms for Tumor-Specific Self-Assembly and Synergistic Therapy
}

\author{
Wei Tang ${ }^{\dagger}$, Wenpei Fan ${ }^{\star}, \dagger$, Zhantong Wang ${ }^{\dagger}$, Weizhong Zhang ${ }^{\ddagger}$, Shiyi Zhou ${ }^{\ddagger}$, Yijing Liu ${ }^{\dagger}$, \\ Zhen Yang ${ }^{\dagger}$, Emily Shao ${ }^{\dagger}$, Guofeng Zhang ${ }^{\S}$, Orit Jacobson ${ }^{\dagger}$, Lingling Shan ${ }^{\dagger}$, Rui Tian ${ }^{\dagger}$, \\ Siyuan Cheng ${ }^{\dagger}$, Lisen Lin ${ }^{\dagger}$, Yulun Dai ${ }^{\dagger}$, Zheyu Shen ${ }^{\dagger}$, Gang Niu ${ }^{\dagger}, \mathrm{Jin} \mathrm{Xie}^{*}, \neq$, and Xiaoyuan \\ Chen ${ }^{*} \dagger$ \\ tLaboratory of Molecular Imaging and Nanomedicine (LOMIN), National Institute of Biomedical \\ Imaging and Bioengineering (NIBIB), National Institutes of Health (NIH), Bethesda, Maryland \\ 20892, United States \\ ¥Department of Chemistry, University of Georgia, Athens, Georgia 30602, United States \\ \&Laboratory of Cellular Imaging and Macromolecular Biophysics, National Institute of Biomedical \\ Imaging and Bioengineering (NIBIB), National Institutes of Health (NIH), Bethesda, Maryland \\ 20892, United States
}

\section{Abstract}

Featured with a large surface area, uniform interpenetrating mesopores, diverse organic framework hybridization, and well-defined surface properties, the hollow mesoporous organosilica nanoparticle (HMON) represents a promising paradigm in drug delivery systems with excellent biocompatibility. However, effective tumor accumulation and precise cancer theranostics of the HMON still remain a challenge. In this study, an "ammonia-assisted hot water etching" method is applied for the successful construction of sub-50 nm thioether/ phenylene dual-hybridized HMON with low hemolytic effect. Particularly, the surface modification with Mo(VI)-based polyoxometalate (POM) clusters drives the self-assembly of HMON in the mild acidic tumor microenvironment (TME) to achieve enhanced tumor retention and accumulation. More importantly, the reducibility-activated $\mathrm{Mo}(\mathrm{VI})$-to-Mo(V) conversion within POM not only endows the POM-anchored HMON with outstanding TME-responsive photoacoustic (PA) imaging contrast and photothermal therapy (PTT) performance but also plays an indispensable role in controllably triggering the decomposition of the $\mathrm{Mn}_{2}(\mathrm{CO})_{10}$ payload for $\mathrm{CO}$ release, which gives rise to remarkable synergistic PTT-enhanced CO gas therapy for complete tumor eradication. By harnessing the unique acidic and redox properties of TME, the judiciously designed smart POManchored HMON nanoplatform is expected to act as a "magic bomb" to selectively destroy cancer

\footnotetext{
*Corresponding Authors: wenpei.fan@nih.gov. jinxie@uga.edu. shawn.chen@nih.gov. ASSOCIATED CONTENT

Supporting Information

The Supporting Information is available free of charge on the ACS Publications website at DOI: 10.1021/acsnano.8b06058. Additional experimental methods and data (Figures S1-S29) (PDF)

Notes

The authors declare no competing financial interest.
} 
without damaging normal tissues. This nanoplatform holds significant potential in realizing TMEresponsive self-assembly for enhanced tumor accumulation and precise tumor-specific synergistic therapy, which is very promising for clinical translation.

\section{Keywords}

hollow mesoporous organosilica nanoparticle; dual hybridization; self-assembly; tumor microenvironment responsiveness; synergistic therapy

As one of the most important branches of mesoporous nanomaterials, mesoporous silica nanoparticles (MSNs) have gone through decades of development since their inception in the 1990s. ${ }^{1,2}$ Prominent characteristics of MSNs, including a large surface area, uniform interpenetrating mesopores, high chemical stability, and controllable surface modification, endow MSNs with a variety of applications in a broad spectrum of fields, especially in biomedicine. ${ }^{3-5}$ To reduce the systemic toxicity accompanied by conventional chemotherapy, biocompatible hollow-structured MSNs (HMSNs) featured with a large loading capacity have been widely used for tumor-specific drug delivery. ${ }^{6,7}$ However, the inert $\mathrm{Si}-\mathrm{O}-\mathrm{Si}$ framework associated with poor biodegradation remains a major concern for clinical use of the HMSNs. ${ }^{8}$ As a result, current research has become increasingly focused on hollow mesoporous organosilica nanoparticles (HMONs). The framework hybridization of diverse functional organic moieties brings about intriguing physiochemical merits. ${ }^{9-12}$ For example, the disulfide-hybridized HMONs are able to gradually degrade in the reductive tumor microenvironment (TME), resulting in glutathione (GSH)-responsive drug release and in vivo clearance. ${ }^{13}$ Additionally, the phenylene-bridged organosilica framework can significantly lower the hemolytic activity of the HMONs for enhanced biocompatibility. ${ }^{14}$ Therefore, the single and multiple organic moiety-hybridized HMONs exhibit unparalleled advantages in stimuli-responsive degradation, controlled drug release, and enhanced biosafety, thus promising significant potential in biomedicine.

Like other drug delivery systems (DDSs), a major concern about HMONs is still the offtarget drug delivery for unavoidably adverse effects on normal tissues. This issue can be partially mitigated by imparting a tumor targeting ligand to the nanoparticle surface. ${ }^{15-18}$ However, the cumbersome conjugation and purification procedures often cause reduced particle stability and batch-to-batch variability. ${ }^{19-23}$ Despite the substantial investment of time, money, and manpower, the efficiency of the active targeting approach remains suboptimal. Therefore, many, including us, have revisited the passive targeting strategy. ${ }^{24}$ Proverbially, nanoparticles with relatively long circulation half-lives can extravasate at leaky tumor vasculatures. ${ }^{25,26}$ To this end, the size of the HMONs should be kept below $50 \mathrm{~nm}$ to minimize clearance by Kupffer cells/ macrophages in the liver and spleen and prolong blood circulation. ${ }^{27-29}$ On the other hand, small nanoparticles are more susceptible to clear from the interstitial space by lymphatic drainage and extravasation, which leads to short tumor retention. To solve the dilemma, some have exploited nanostructures that maintain individually dispersed in the blood circulation but self-assemble into much large aggregates once entering the TME, ${ }^{30,31}$ for instance, the "acidity-triggered self-assembly" of molybdenum-based polyoxometalate (POM) clusters. ${ }^{31}$ 
In this study, we aim to leverage the POM strategy to achieve smart sub-50 nm HMONs that can self-assemble within tumors for enhanced accumulation and retention. We propose a special "ammonia-assisted hot water etching" method, which allows for a controllable synthesis of sub-50 nm dual-hybridized HMONs through the concurrent introduction of two kinds of bissilylated organosilica precursors. The framework hybridization of thioether and phenylene renders the HMONs much less hemolysis than HMSNs. Moreover, the dual hybridization allows for efficient encapsulation of hydrophobic guest molecules into the HMONs. ${ }^{32}$ In the current study, a typical paradigm of CO-releasing molecule, $\mathrm{Mn}_{2}(\mathrm{CO})_{10}$, is loaded into the HMONs through hydrophobic-hydrophobic interactions. Ultrasmall POM clusters are attached to the surface of HMONs via the metal-thiol coordination chemistry. 33,34 It is postulated that the acidic tumor microenvironment (TME) will cause protonation of POM, leading to aggregation of HMONs. Meanwhile, the reductive TME will induce $\mathrm{Mo}(\mathrm{VI})$-to-Mo(V) reduction and, associated with it, reproduce strong near-infrared (NIR) absorption, which favors photoacoustic (PA) imaging as well as photothermal therapy (PTT). PTT can further trigger the thermal decomposition of the $\mathrm{Mn}_{2}(\mathrm{CO})_{10}$ payload, releasing CO that synergistically works with PTT for enhanced cancer treatment (Scheme 1). Such acidity/reducibility dual-responsive HMON nanoplatforms are expected to achieve more enhanced tumor accumulation and precise treatment by taking advantage of the unique acidic and GSH-enriched TME, representing a paradigm design of tumor-specific cancer theranostics with minimal side effects on normal tissues.

\section{RESULTS AND DISCUSSION}

\section{Design, Synthesis, and Characterization of Sub-50 nm Thioether/Phenylene Dual- Hybridized HMONs.}

Smallsized HMONs are difficult to prepare by conventional methods which are suboptimal in control of particle size, shell thickness, and etching rate. Herein, this issue was solved by a unique "ammonia-assisted hot water etching" approach on the basis of the "chemical homology" principle. As shown in Figure 1a, monodispersed $30 \mathrm{~nm}$ MSNs were first synthesized under structural direction of cetyltrimethylammonium chloride (CTAC) and alkaline catalysis of triethanolamine (TEA) (Figure 1b and S1a). Next, through cohydrolysis and co-condensation of an equal volume mixture of bis(triethoxysilyl)-phenylene (BTEB, $\mathrm{R}_{1}$ $=$ phenylene) and bis[3-(triethoxysilyl)-propyl]tetrasulfide (BTES, $\mathrm{R}_{2}=$ thioether), a thin shell of MONs with framework hybridization of phenylene and thioether were successfully coated onto the surface of MSN templates. The resulting MSN@MON nanostructure had an average particle size of around $40 \mathrm{~nm}$ (Figures $1 \mathrm{~b}$ and S1b). Finally, ammonia was employed as a mild etching agent to carve out the cavity with good control. During the process, the inorganic MSN core was selectively removed since $\mathrm{Si}-\mathrm{C}$ bonds are much more resistant to alkaline etching than $\mathrm{Si}-\mathrm{O}$ bonds, leaving behind thioether/phenylene hybridized HMONs.

Transmission electron microscopy (TEM) images found that the HMONs are uniform and well-dispersed hollow spheres, with an average size of around $40 \mathrm{~nm}$ and a thin shell of around $5 \mathrm{~nm}$ (Figures 1b and S1c). The hydrodynamic sizes of MSN, MSN@MON, and HMON were determined to be $60.5 \pm 6.2,76.0 \pm 7.3$, and $73.3 \pm 8.6 \mathrm{~nm}$, respectively (Figure S2). The Raman spectrum of HMON displayed stretching vibrations of $-\mathrm{S}-\mathrm{S}-$ bond 
at $438 / 488 \mathrm{~cm}^{-1}$ and $-\mathrm{S}-\mathrm{C}-$ bond at $636 \mathrm{~cm}^{-1}$ (Figure 1c), confirming the incorporation of the thioether moiety within the framework. The energy dispersive X-ray spectroscopy (EDS) spectrum further validated the successful thioether/phenylene hybridization for finding the characteristic peaks of the $\mathrm{Si}, \mathrm{C}, \mathrm{O}$, and $\mathrm{S}$ (Figure 1d). The dual-hybridized HMONs exhibited well-defined mesoporous structures with a large surface area of $475.2 \mathrm{~m}^{2} / \mathrm{g}$ (Figure 1e) and a pore size of around $4.3 \mathrm{~nm}$ (Figure 1f). The internal cavity allowed for efficient encapsulation of diverse types of cargos. Importantly, the well-designed dual-hybridized HMONs showed remarkably reduced hemolytic effects against red blood cells (RBCs) compared to the traditional HMSNs, which implied high biosafety of HMONs in the blood circulation system after intravenous injection (Figure 1g and S3). As shown in Figure 1g, the hemolytic percentage of HMON-treated RBCs was only $7.6 \%$ at $250 \mu \mathrm{g} / \mathrm{mL}$, while almost all RBCs were hemolyzed by conventional HMSNs at this concentration. The reduced hemolysis was attributed to the organic hybridization. By introducing an inert phenylene group into a silica matrix, the amount of surface-exposed silanol groups $(\mathrm{Si}-\mathrm{OH})$ that is known to induce hemolysis ${ }^{32,35,36}$ was dramatically decreased, leading to improved biocompatibility.

\section{Design, Synthesis, and Characterization of a Tumor Microenvironment (TME)-Responsive Self-Assembled HMON Nanoplatform.}

To develop a TME-responsive self-assembled HMON nanoplatform, Mo-based POM was anchored onto the HMON surface (Figure 2a). Briefly, POM was synthesized via a facile, fast, and large-scale synthesis process. ${ }^{31,37}$ The assynthesized HMONs reacted with both silane-PEG and (3-mercaptopropyl)trimethoxysilane (MPTES), which made the particles both PEGylated and thiolated. After surface functionalization, the POMs were anchored onto the HMON surface through strong molybde-num-thiol binding. Elemental mapping (Figure 2b) found that PEG and POM cofunctionalized HMONs (HMONs-PEG/ POM, denoted as HMOPMs) retained a hollow nanostructure, with Mo homogeneously deposited on the shell. EDS analysis (Figure 2c) further confirmed the successful deposition of Mocontaining POM onto the particle surface. X-ray photoelectron spectroscopy (XPS) (Figure 2d) found that all the Mo elements were in one oxidation state consistent with $\mathrm{Mo}(\mathrm{VI})$, indicating that no unwanted redox reactions happened during the POM functionalization. The Mo content in the HMOPMs was determined to be $6.53 \mathrm{wt} \%$ by inductively coupled plasmaoptical emission spectrometry (ICP-OES).

For POM macroanions, acidification-induced protonation can remarkably decrease the electrostatic repulsion between adjacent units and simultaneously increase the short-range attractive forces through hydrogen bonding. ${ }^{31,38}$ It was speculated that the surface-modified POM would be protonated in an acidic environment and drive the HMOPMs to form large assemblies via the intermolecular hydrogen bonding of POM (Figure 2e). To test our hypothesis, we dispersed the HMOPMs in solutions with different $\mathrm{pH}$ values. TEM images showed that the HMOPMs remained colloidally stable at $\mathrm{pH}$ 7.4, but self-assembled into micrometer-scaled clusters under slightly acidic conditions $(\mathrm{pH}=6.5)$ and aggregated into even larger structures with further acidification to $\mathrm{pH} 5.5$ (Figure 2f). The hydrodynamic size of the HMOPMs correspondingly increased from $106.1 \pm 11.1 \mathrm{~nm}$ at $\mathrm{pH}=7.4$ to 1367.5 \pm 272.7 and $3568.4 \pm 891.8 \mathrm{~nm}$ at $\mathrm{pH} 6.5$ and 5.5, respectively (Figure 2g). The successful 
acidity-driven aggregation was further confirmed by a higher magnification TEM image and EDS spectrum of HMOPM at pH 5.5 (Figure S4). In the large aggregates, the morphology of HMOPM remained the same as that in $\mathrm{pH} 7.4$ solution, showing intact and rigid hollow sphere structures. Moreover, longitudinal DLS measurements with successive acidification demonstrated excellent colloidal stability of HMOPM at neutral $\mathrm{pH}$ and formation of stable large assemblies in acidic environments (Figure S5). In contrast, successive acidification induced no size changes of the PEG and thiol cofunctionalized HMON (HMON-PEG/-SH) control (Figure S5), indicating excellent colloidal stability of HMON-PEG/-SH in response to acidity and steady POM modification in HMOPM. The aggregation of HMOPM was again attributed to the hydrogen bonds formed between the terminal oxygen or edge-sharing oxygen in the POM and the proton from the acidification.

\section{Acidity/Reducibility Dual-Responsive Photothermal Conversion and Photoacoustic (PA) Contrast of the HMOPMs.}

Interestingly, the color of the HMOPM solutions was observed to change from colorless to blue and then monotonically deepen with an increasing concentration of GSH (Figure 3a). This was consistent with enhanced NIR absorption detected by a UV-vis spectrometer (Figures $3 \mathrm{~b}$ and S6a). In addition, increasing the acidity of the GSH solutions led to a darker blue color and further enhanced NIR absorption (Figures $3 b$ and S6b). The intensified NIR absorption was attributable to the occupiable cation site of $\mathrm{Mo}(\mathrm{V})$ (Figure S7), and both the reduction and acidification-promoted electron density delocalization in the POM. The NIR absorption could be further strengthened through electron relaxation polarization under an external electromagnetic field, such as laser light, ${ }^{31,37}$ leading to excellent NIR photothermal conversion and photoacoustic contrast performance. This inspired us to investigate the photothermal conversion and PA contrast abilities of HMOPMs and their dependence on acidity and reducibility. For photo-thermal conversion, HMOPM solutions with varied $\mathrm{pH}$ and GSH concentrations were irradiated with an NIR laser $(808 \mathrm{~nm}, 1$ $\mathrm{W} / \mathrm{cm}^{2}$ for $5 \mathrm{~min}$ ) and their temperature changes were monitored by an infrared (IR) camera. The IR photographs revealed a distinctive $\mathrm{pH}$ - and GSH-dependent photothermal effect (Figure 3c). The GSH-reduced HMOPMs showed a rapid temperature rise in the first minute of the irradiation and gradually reached a plateau. For $\mathrm{pH} 7.4$ solutions, at the end of irradiation, the temperature was increased by $0.7,20.1,31.1,41.6$, and $45.6{ }^{\circ} \mathrm{C}$ when the GSH concentration was $0,2,5,10$, and $20 \mathrm{mM}$, respectively. For pH 6.5 and 5.5 solutions, the corresponding temperature increases were $0.5,25.7,36.3,44.4,47.2^{\circ} \mathrm{C}$ and $0.2,33.3$, $43.3,48.8,50.9^{\circ} \mathrm{C}$, respectively (Figures $3 \mathrm{~d}$ and S8). These observations suggested that HMOPMs at the highest oxidation state of Mo(VI) showed no photothermal effect, but after reduction by GSH, they exhibited more efficient photothermal conversion in solutions with higher acidity and, more prominently, with stronger reducibility. PA imaging results (Figure $3 e$ ) and quantitative analysis (Figure 3f) showed a similar result. It is worth noting that the HMOPM remained stable in a reductive environment for at least 3 days (Figure S9). The enhanced stiffness of the HMOPM framework might be attributed to the phenylene group incorporation. The acidity-driven self-assembly could also be observed in a reductive environment, indicating excellent stability of Mothiol binding (Figure S10). 
The above observations suggested the great potential of HMOPMs as a tumor selective theranostic agent. It was reasoned that upon activation by the reductive TME, HMOPM would initiate charge transfer between $\mathrm{Mo}(\mathrm{VI})$ and $\mathrm{Mo}(\mathrm{V})$ through the bridging oxygen bonds in POM, leading to the delocalization of the electron density and the occupation of the $\mathrm{Mo}(\mathrm{V})$ cation site through reversible multistep electron exchanges (Figure 3g). ${ }^{31,37}$ Meanwhile, the acidic TME would cause protonation of POM's edge-sharing oxygen atoms, further facilitating electron delocalization by broadening the gap between the highest occupied molecular orbital (HOMO) and the lowest unoccupied molecular orbital (LUMO). 31,37 These effects translated into photothermal and PA contrast abilities, which were only prominent in tumors.

\section{Enhanced Tumor Accumulation Based on the Acidity-Driven HMOPM Self-Assembly.}

The acidity-driven selfassembly of HMOPMs also indicated enhanced tumor retention through in situ size expansion within TME. This was first investigated in vitro with U87MG cells. The cells were incubated with HMOPMs for $24 \mathrm{~h}$ and then subjected to bio-TEM analysis (Figure 4a). Many HMOPM aggregates were detected in the cell endosomes ( $\mathrm{pH}$ $5-6) .{ }^{39,40}$ In contrast, the HMON control group (i.e., without surface modification of POM) did not exhibit obvious assemblies. Moreover, the EDS spectra (Figure 4b) found the presence of Mo and an enhanced level of $\mathrm{Si}$ in the HMOPM-treated cells. These observations consolidated that POM was the main driving force behind the HMOPM selfassembly and the acidic environment as the trigger. Notably the HMOPMs and their constituent components (i.e., POMs and HMONs) all showed good biocompatibility in vitro (Figure S11).

To investigate whether the acidity-driven aggregation of HMOPMs would contribute to enhanced tumor accumulation, ${ }^{64} \mathrm{Cu}$-labeled HMOPMs and HMONs were intravenously injected into U87MG tumor bearing mice and migration of the nanoparticles was monitored by positron emission tomography (PET) imaging (Figure $4 \mathrm{c}$ ). The ${ }^{64} \mathrm{Cu}$ labeling exhibited excellent stability in phosphate-buffered saline (PBS) (Figure S12). The HMOPMs accumulated in tumors in $1 \mathrm{~h}$, and the tumor accumulation was ever increased, reaching 7.39 $\pm 0.92 \% \mathrm{ID} / \mathrm{g}$ at $24 \mathrm{~h}$ postinjection (p.i.). At all examined time points, HMOPMs displayed a much higher tumor uptake than HMONs, with an increase by 2.21-, 2.05-, 1.76-, and 1.37fold at 1, 4, 24, and $48 \mathrm{~h}$ p.i., respectively (Figure $4 \mathrm{~d}$ ). The enhanced uptake was attributed to TME induced nanoparticle self-assembly and thereby improved tumor retention. Interestingly, the liver and spleen uptake of HMOPMs was strikingly decreased relative to HMONs (Figure 4e and 4f). The mechanism behind the decreased uptake is unknown and worth further investigation. After the imaging, tumors and major organs were collected for radioactivity measurement by gamma countering (Figure $4 \mathrm{~g}$ ). Consistent with the in vivo PET imaging results, the tumor uptake of the HMOPMs was increased by $152 \%$ relative to HMONs, and the liver and spleen uptake decreased by $38.35 \%$ and $54.03 \%$, respectively.

\section{TME-Responsive and Photothermal-Controlled CO Release.}

Instead of chemotherapeutics, a typical CO-releasing molecule, $\mathrm{Mn}_{2}(\mathrm{CO})_{10}$, was loaded into the cavity of HMOPMs (Figure $5 \mathrm{a}$ ). $\mathrm{Mn}_{2}(\mathrm{CO})_{10}$ was first encapsulated into HMONs via hydrophobic-hydrophobic interactions. Subsequently, POM was coated onto the surface of 
HMONs, forming $\mathrm{Mn}_{2}(\mathrm{CO})_{10} @$ HMOPMs (denoted as HMOPMs-CO). The TEM found a hollow sphere structure with a diameter of around $50 \mathrm{~nm}$ (Figure 5b). The loading capacity of Mn was about $1 \mathrm{wt} \%$ based on ICP analysis. Elemental mapping of HMOPMs-CO (Figure 5c) showed homogeneous distribution of both Mo and Mn in the nanostructure. EDS analysis (Figure 5d) further confirmed the presence of Mo and Mn elements. The HMOPMsCO showed a comparable surface area of $491.84 \mathrm{~m}^{2} / \mathrm{g}$ to HMONs (Figure $5 \mathrm{e}$ ) but a reduced pore size ( $\sim 3.7 \mathrm{~nm}$, Figure $5 \mathrm{f}$ ), suggesting that some of the $\mathrm{Mn}_{2}(\mathrm{CO})_{10}$ molecules were loaded into the channels of the HMONs. The HMOPMs-CO also exhibited a similar aciditytriggered aggregation behavior, evidenced by an increased hydrodynamic size from 124.7 $\pm 13.7 \mathrm{~nm}$ to $1467.4 \pm 285$ and $4781.5 \pm 882.6 \mathrm{~nm}$ when the $\mathrm{pH}$ was lowered from 7.4 to 6.5 and 5.5, respectively (Figure S13). The XPS deconvoluted spectra for Mo 3d orbitals of the HMOPMs-CO (Figure 5g) were in good correlation with the peaks of HMOPMs (Figure $2 \mathrm{~d}$ ), indicating that the $\mathrm{Mn}_{2}(\mathrm{CO})_{10}$ loading had little impact on POM modification. Based on these observations, we postulated that the TME induced nanoparticle self-assembly and photothermal conversion would still apply for HMOPMs-CO. To confirm it, HMOPMs-CO were incubated in solutions containing $10 \mathrm{mM} \mathrm{GSH}$ and irradiated by an $808 \mathrm{~nm}$ laser at elevated fluence rates $\left(0.3,0.5,1.0 \mathrm{~W} / \mathrm{cm}^{2}\right)$ for $20 \mathrm{~min}$ (Figure $5 \mathrm{~h}$ ). At all fluences, temperature was rapidly increased in the first $3 \mathrm{~min}$ of irradiation and plateaued after about 5 $\min$. The increase amplitude was positively correlated with the irradiation fluence, with the final temperature being $40.1,51.8$, and $68.8^{\circ} \mathrm{C}$ for $0.3,0.5$, and $1.0 \mathrm{~W} / \mathrm{cm}^{2}$, respectively.

It has been reported that the photothermal effect could induce the cleavage of the metal CO bond and that the $\mathrm{CO}$ release rate could be controlled by adjusting irradiation intensity and duration. ${ }^{41}$ To test whether this applied for our system, we monitored CO release from HMOPMs-CO using COP-1, a green fluorogenic $\mathrm{CO}$ indicator, ${ }^{42}$ under varied irradiation conditions. As shown in Figure 5i, the CO release was dependent on both irradiation intensity and duration. For example, a similar amount of $\mathrm{CO}$ was released upon exposure to laser of $0.3 \mathrm{~W} / \mathrm{cm}^{2}$ for $12 \mathrm{~min}$ or $1.0 \mathrm{~W} / \mathrm{cm}^{2}$ for $3 \mathrm{~min}$. Note that $0.3 \mathrm{~W} / \mathrm{cm}^{2}$ could only heat the solution to a mild hyperthermia temperature of around $40^{\circ} \mathrm{C}$, while the high fluence rate of $1.0 \mathrm{~W} / \mathrm{cm}^{2}$ could rapidly heat the solution to $70{ }^{\circ} \mathrm{C}$ (Figure $5 \mathrm{~h}$ ). For the subsequent in vitro and in vivo therapy studies, two irradiation conditions would be investigated: 0.3 $\mathrm{W} / \mathrm{cm}^{2}$ to induce CO release but sublethal PTT effects, and $1.0 \mathrm{~W} / \mathrm{cm}^{2}$ to result in both CO release and photothermal ablation.

It is well-known that $\mathrm{CO}$ exerts broad biological effects on inflammation, apoptosis, and cellular proliferation, ${ }^{43}$ where the cell mitochondria and the overall bioenergetics are a central target. ${ }^{43,44}$ Exposure to $\mathrm{CO}$ drives cancer cells to consume more oxygen for energy supply, causing accelerated biogenesis, mitochondria exhaustion, elevated reactive oxygen species (ROS) production, and eventually cell apoptosis. ${ }^{43-45}$ It was expected that CO released from HMOPMs-CO could result in cell death. To test the hypothesis, U87MG cells were incubated with HMOPMs-CO (preincubated with GSH) and irradiated by an $808 \mathrm{~nm}$ laser at elevated fluences $\left(0.3,0.5,1.0 \mathrm{~W} / \mathrm{cm}^{2}\right)$ for $3 \mathrm{~min}$. Significant $\mathrm{CO}$ release was detected by monitoring COP-1 fluorescence under a confocal microscope (Figures 6a and $\mathrm{S} 14 \mathrm{a})$. In particular, cells exposed to a $0.3 \mathrm{~W} / \mathrm{cm}^{2}$ irradiation for $12 \mathrm{~min}$ displayed a comparable level of positive COP-1 staining to those treated with a $1.0 \mathrm{~W} / \mathrm{cm}^{2}$ irradiation for $3 \mathrm{~min}$. Similar results were observed in flow cytometry, finding a relatively low 
percentage of positive staining (11.8\%) in cells treated with $0.3 \mathrm{~W} / \mathrm{cm}^{2}$ for $3 \mathrm{~min}$, but $100 \%$ positive staining in cells treated with $1.0 \mathrm{~W} / \mathrm{cm}^{2}$ for $3 \mathrm{~min}$ or $0.3 \mathrm{~W} / \mathrm{cm}^{2}$ for $12 \mathrm{~min}$ (Figure $\mathrm{S} 14 \mathrm{~b}$ ). These observations correlated well with those made in solutions (Figure 5i), indicating that the NIR irradiation could precisely control CO release from HMOPMs-CO even in vitro.

Next, JC-1 assay was performed to assess the impact of the released $\mathrm{CO}$ on mitochondria. JC-1 forms J-aggregates with intense red fluorescence in healthy cells (with high mitochondrial membrane potential) but remains in the monomeric form with green fluorescence in apoptotic cells (with low mitochondrial membrane potential). ${ }^{45,46} \mathrm{We}$ incubated U87MG cells with the HMOPMs-CO (100 $\mu \mathrm{g} \mathrm{Mo} / \mathrm{mL}$, preincubated with GSH) and irradiated the cells with an $808 \mathrm{~nm}$ laser. The irradiation dose was set at $0.3 \mathrm{~W} / \mathrm{cm}^{2}$ for 12 min to minimize the photothermal effect on the cancer cells. HMOPMs-CO alone (100 $\mu \mathrm{g} \mathrm{Mo} / \mathrm{mL})$, irradiation alone $\left(0.3 \mathrm{~W} / \mathrm{cm}^{2}\right.$ for $\left.12 \mathrm{~min}\right)$, and PBS only were studied as controls. HMOPMs-CO exhibited excellent biocompatibility toward U87MG cells in the dark (Figure S15). Confocal fluorescence imaging observed a significant decrease in the red (JC-1 aggregates) to green (JC-1 monomer) ratio in the HMOPMs-CO plus NIR group (Figures $6 \mathrm{~b}$ and S16a), indicating depolarized mitochondria. In sharp contrast, all control groups exhibited negligible green florescence. Flow cytometry analysis (Figures $6 \mathrm{c}$ and S16b) verified the results, finding a $44.0 \pm 3.7 \%$ loss in mitochondrial membrane potential after irradiation along with the HMOPMs-CO, but insignificant decrease in aggregate fluorescent counts in all controls. In addition, HMOPM plus NIR did not damage mitochondria membranes, indicating the depolarized mitochondria in the HMOPMs-CO plus NIR group resulted from the photothermal-induced CO release but not the mild photothermal effect (Figure S17). Moreover, the intracellular ROS level was detected using a 2', $7^{\prime}$-dichlorodihydrofluorescein diacetate $\left(\mathrm{H}_{2}\right.$ DCFDA) probe by both confocal microscopy (Figures 6d and S18) and flow cytometry (Figure 6e). A drastic increase of ROS generation was found in cells treated with the HMOPMs-CO plus irradiation, whereas limited green fluorescence was found in the controls. All these observations support the hypothesis that NIR-triggered CO release from the HMOPMs-CO could cause mitochondrial exhaustion and ROS generation, leading to cell death. ${ }^{43,45}$

\section{Synergistic Therapy by PTT and CO in Vitro.}

The synergy between PTT and CO therapy was investigated in U87MG cells by MTT assays (Figure 7a). Cells were treated with HMOPMs-CO $(100 \mu \mathrm{g} \mathrm{Mo} / \mathrm{mL}$, preincubated with GSH) and exposed to an $808 \mathrm{~nm}$ irradiation. When irradiated at $1.0 \mathrm{~W} / \mathrm{cm}^{2}$ for $3 \mathrm{~min}$ (denoted as G1 group, where cells were expected to receive PTT and CO combinational therapy), cell viability dropped to $30.1 \pm 3.2 \%$. This was significantly lower than 48.1 $\pm 4.7 \%$ observed in the PTT only group (denoted as G2, HMOPMs plus $1.0 \mathrm{~W} / \mathrm{cm}^{2}$ for 3 $\mathrm{min}$ ) and $65.9 \pm 5.4 \%$ in the CO only group (G3, HMOPMs-CO plus $0.3 \mathrm{~W} / \mathrm{cm}^{2}$ for 12 $\mathrm{min})$. Notably, only a marginal cell viability drop was observed in the mild hyperthermia group (G4, HMOPMs plus $0.3 \mathrm{~W} / \mathrm{cm}^{2}$ for $12 \mathrm{~min}$ ), the HMOPMs-CO only group (G5), and the irradiation only group $\left(\mathrm{G} 6,1.0 \mathrm{~W} / \mathrm{cm}^{2}\right.$ for $\left.3 \mathrm{~min}\right)$. The $f_{\mathrm{CO}+\mathrm{PTT}}$ is less than the $f_{\text {additive }}$ $\left(=f_{\mathrm{CO}} \times f_{\mathrm{PTT}}\right)$, where $\mathrm{f}$ is the fraction of viable cells after each treatment, demonstrating the synergistic effect of PTT and CO therapy. ${ }^{47}$ 
Cell viability was also investigated by live/dead assays with Calcein-AM and propidium iodide (PI) double staining (Figure 7b). Similar to the MTT results, we observed negligible positive PI staining in the mild hyperthermia (G4), HMOPMs-CO (G5), NIR irradiation (G6), and PBS (G7) groups. As a comparison, in the synergistic PTT enhanced CO therapy group (G1), almost all the cells were positively stained by PI. Flow cytometry (Figure 7c and 7d) further verified these observations. In addition, without preincubation with GSH, the HMOPMs-CO and the HMOPMs showed little phototoxicity (Figure S19), implying that reduction activation was a precondition for effective treatment. The combination of PTT and CO led to more significant cell killing, which may be attributed to their synergistic enhancement interactions. The photothermal effect could not only directly kill cancer cells but also promote the cellular uptake of the $\mathrm{HMOPMs}-\mathrm{CO}^{48}$ and accelerate the $\mathrm{CO}$ release, ${ }^{41}$ therefore remarkably enhancing the $\mathrm{CO}$ treatment efficacy.

\section{In Vivo TME-Responsive PA and PTT of the HMOPMs-CO.}

The in vivo biosafety was assessed in balb/c mice. Seven days after the intravenous injection of HMOPMs-CO or HMOPMs (10 mg Mo/kg), the mice were sacrificed, blood was drawn for blood index tests, and major organs were harvested for histological analysis. The PBS was tested as a control. The complete blood count (CBC) parameters, including white blood cells (WBC), red blood cells (RBC), hemoglobin (HGB), hematocrit (HCT), mean corpuscular volume (MCV), and platelets (PLT), showed no obvious changes (Figure S20a), indicating that both the HMOPMs-CO and the HMOPMs caused no significant inflammation or infection. Furthermore, liver functional indexes such as alanine transaminase (ALT) and aspartate transaminase (AST), renal functional biomarker blood urea nitrogen (BUN), and other metabolic parameters such as glucose (GLU), total protein (TP), and albumin (ALB) were measured (Figure S20b). Compared to the PBS control, no abnormalities were found in the two nanoparticle-treated groups, suggesting negligible toxicity. H\&E staining on heart, liver, spleen, lung, and kidneys found no apparent acute pathological changes in all groups (Figure S21). In addition, no obvious mouse body weight drop was observed throughout the study (Figure S22). These results demonstrated the relatively high in vivo biocompatibility of the HMOPMs-CO and the HMOPMs. To make it a more clinically translational nanotheranostic agent, further optimizations on the HMOPM nanoparticle could be done. For example, modulating its surface chemistry to further increase the in vivo stability, reducing the nanoparticle size to enhance its passive tumor accumulation by the EPR effect, modifying the particle with active targeting ligands to increase its tumor homing, and increasing the Mo loading rate to enhance its TMEresponsive aggregation for precise tumor imaging and treatment.

Next, TME-responsive PA and PTT were examined in vivo. After intravenous injection of the HMONPM-COs into U87MG tumor-bearing mice, the PA signals at tumors were gradually increased at early time points, reaching the maximum intensity at $24 \mathrm{~h}$ p.i. and maintaining a high contrast through 48 h p.i. (Figure 8a). Mice treated with the HMOPMs at the same dosage showed similar PA contrast changes in tumors (Figure 8a), which was not surprising because HMOPMs-CO and HMOPMs shared the same feature of TME-activated PA contrast enhancement. Interestingly, although both exhibited the maximum contrast at 24 h p.i., the PA signal changes in the HMOPM group were slightly different from the PET 
results. PET imaging (Figure $4 \mathrm{c}$ and $4 \mathrm{~d}$ ) revealed an obvious tumor accumulation of the HMOPMs as early as at $1 \mathrm{~h}$ p.i. and a relatively high accumulation at $4 \mathrm{~h}$ p.i. However, the PA contrast was not easily detected until $8 \mathrm{~h}$ p.i., which was attributable to the reducibility and acidity dual-responsive PA contrast of the HMOPM components. It took time for the $\mathrm{Mo}(\mathrm{VI})$-to-Mo(V) reduction to occur, which was why the PA signals were not immediately enhanced upon tumor uptake.

The HMOPMs-CO and the HMOPMs also went through a self-adaptive photothermal conversion in vivo. Guided by PA imaging, a laser was applied to tumors at $24 \mathrm{~h}$ p.i. Two irradiation dosages were applied to evaluate the PTT effects: a high fluence rate of 1.0 $\mathrm{W} / \mathrm{cm}^{2}$ for $5 \mathrm{~min}$ and a comparatively low fluence rate of $0.3 \mathrm{~W} / \mathrm{cm}^{2}$ for $20 \mathrm{~min}$. These two conditions should induce the same level of $\mathrm{CO}$ release as shown in Figure 5i. The temperature of tumors treated with both the HMOPMs-CO and the HMOPMs was rapidly increased to about $47{ }^{\circ} \mathrm{C}$ within 2 min and maintained at around $49^{\circ} \mathrm{C}$ upon the $1.0 \mathrm{~W} / \mathrm{cm}^{2}$ irradiation, whereas the $0.3 \mathrm{~W} / \mathrm{cm}^{2}$ irradiation could only heat the tumors to a temperature below $40{ }^{\circ} \mathrm{C}$ (Figures 8b, 8c, 8d, and S23). Besides, tumors treated with PBS showed little temperature changes upon $808 \mathrm{~nm}$ irradiation at either $1.0 \mathrm{~W} / \mathrm{cm}^{2}$ or $0.3 \mathrm{~W} / \mathrm{cm}^{2}$. As such, HMOPMs plus a $1.0 \mathrm{~W} / \mathrm{cm}^{2} \mathrm{NIR}$ irradiation could indeed produce remarkable photothermal-killing effects in vivo.

\section{In Vivo Synergistic Photothermal-Enhanced CO Therapy with the HMOPMs-CO.}

In vivo therapy studies were performed in U87MG tumor models ( $\mathrm{n}=5 /$ group). The mice were systematically administrated with HMOPMs-CO $(10 \mathrm{mg} \mathrm{Mo} / \mathrm{kg})$, followed by an 808 $\mathrm{nm}$ irradiation at $1.0 \mathrm{~W} / \mathrm{cm}^{2}$ for $5 \mathrm{~min}$ to tumors at $24 \mathrm{~h}$ p.i. (G1). As shown in Figure 8e and 8 f, the synergistic PTT/gas therapy induced $100 \%$ tumor eradication on day 4 with no tumor recurrence. In sharp contrast, the PTT alone group (G2) with the HMOPM injection plus a $1.0 \mathrm{~W} / \mathrm{cm}^{2}$ irradiation for $5 \mathrm{~min}$ only revealed a considerable tumor growth inhibition (TGI) rate of $86.3 \pm 1.7 \%$ on day 20 . And the gas therapy alone group (G3) with the HMOPMsCO plus a low irradiation fluence of $0.3 \mathrm{~W} / \mathrm{cm}^{2}$ for 20 min exhibited a TGI of $71.0 \pm 9.1 \%$ on the same day. In addition, a mild hyperthermia group (G4) of HMOPMs plus a 0.3 $\mathrm{W} / \mathrm{cm}^{2}$ irradiation for $20 \mathrm{~min}$ showed marginal tumor inhibition, implying that it was not the mild hyperthermia from the HMOPM components, but the mild hyperthermia-activated $\mathrm{CO}$ release from the HMOPMs-CO that devastated the tumors. In other control groups, including HMOPMs-CO (G5), an NIR irradiation at $1.0 \mathrm{~W} / \mathrm{cm}^{2}$ for $5 \mathrm{~min}$ (G6), and PBS (G7), negligible tumor inhibition was observed. The effective tumor eradication in G1 may result from the synergistic PTT-enhanced gas therapy efficacy. At the beginning of the irradiation, a mild hyperthermia may increase the blood flow and broaden the endothelial gaps in tumors, ${ }^{49,50}$ thus improving the intratumoral delivery of the HMOPMs-CO. As the irradiation went on, the photothermal heating would greatly accelerate the release and diffusion of $\mathrm{CO}$ in the tumors, ${ }^{41}$ which could expose $\mathrm{CO}$ to a much larger scale of tumor cells and further improve the CO-killing effects. The $\mathrm{CO}$ diffusion in the tumor region along with the treatment process was demonstrated by immunofluorescence staining on heme oxygenase-1 (HO-1) after the treatments (Figure S24), whose expression was downregulated by $\mathrm{CO}$ from CO-releasing molecules. ${ }^{51}$ All the above mechanisms accounted for the remarkable synergistic enhancement effect of PTT on CO therapy, leading to complete 
tumor eradication. In addition, to investigate the contribution of the POM component to the synergistic therapeutic efficacy, we studied two other control groups, $\mathrm{Mn}_{2}(\mathrm{CO})_{10}$-loaded and PEG-modified HMON (HMON-CO) or HMON-CO with NIR irradiation (808 nm, $1 \mathrm{~W} /$ $\mathrm{cm}^{2}, 5 \mathrm{~min}$ ) (Figure S25). The two treatment groups showed negligible tumor inhibition compared with the PBS group. The POM could not only induce acidity-driven self-assembly for enhanced tumor retention and accumulation, but also convert light energy into heat. The HMON-CO plus NIR irradiation was not working, as there was a lack of a photothermal conversion agent to induce $\mathrm{CO}$ release as well as photoablation to tumors.

To better differentiate the efficacy of the treatments, tumors were harvested for histological analysis after $24 \mathrm{~h}$ of the treatments. H\&E staining images (Figure S26) found severe structure disruption in the PTT and/or CO treatment groups (G1-G3), especially in the two PTT involved groups (G1 and G2), which also observed large areas of bleeding sites in tumors. TUNEL staining images (Figures $8 \mathrm{~g}$ and S27) demonstrated similar results. A growing level of the positive green fluorescence was visualized in the CO only, PTT only, and combinational PTT and CO treatment groups, indicating an intensified extent of cell apoptosis. Meanwhile, marginal efficacy in the other four control groups was detected based on both H\&E and TUNEL staining results (Figures 8g, S26, and S27). These observations well agreed with the tumor growth curves (Figure 8e) and the tumor inhibition rates (Figure 8f). After the therapy, major organs were acquired for histological analysis, which showed no apparent pathological abnormalities in the H\&E staining images (Figure S28). In addition, no noticeable mice body weight drops were observed during the therapy studies (Figure S29), suggesting little side effects and high safety of these treatments.

\section{CONCLUSIONS}

In summary, we have presented a $\mathrm{pH} / \mathrm{GSH}$ dual stimuli-responsive organosilica-based nanoplatform for tumor-specific self-assembly and synergistic cancer therapy. The welldesigned sub-50 nm thioether/phenylene dual-hybridized HMONs demonstrated low hemolytic effect and high biocompatibility as well as efficient encapsulation of hydrophobic $\mathrm{Mn}_{2}(\mathrm{CO})_{10}$ molecules. Through surface functionalization with Mo(VI)-based POM, the resulting HMOPMs could not only spontaneously self-assemble into large aggregates in the acidic TME for enhanced tumor accumulation via the improved EPR effect but also exhibit excellent GSH-responsive photothermal conversion and PA imaging performance through the reductive TME-driven $\mathrm{Mo}(\mathrm{VI})$-to-Mo(V) reduction. Moreover, the photothermal effect of the HMOPMs could control the $\mathrm{CO}$ release from the $\mathrm{Mn}_{2}(\mathrm{CO})_{10}$ payload through the regulation of NIR irradiation fluence and duration, which bridged the gap between PTT and gas therapy to achieve substantially improved synergistic therapy for tumor eradication. In addition to providing a simple yet effective methodology for enhancing the tumor-specific delivery of nanoparticles based on in situ self-assembly, the excellent marriage of the POMs and the HMONs is also expected to establish an innovative paradigm of TME-responsive theranostic agent for precise cancer synergistic therapy with extremely few side effects. 


\section{METHODS}

Materials.

Cetyltrimethylammonium chloride (CTAC), tetraethyl orthosilicate (TEOS), ammonium hydroxide, triethanolamine (TEA), bis[3-(triethoxysilyl)propyl] tetrasulfide (BTES), dimethyl sulfoxide (DMSO), bis(triethoxysilyl)phenylene (BTEB), (3-mercaptopropyl)trimethoxysilane (MPTES), ammonium molybdate tetrahydrate, sodium phosphate monobasic dihydrate, manganese( 0 ) carbonyl, sodium carbonate, ethanol, glutathione (GSH), thiazolyl blue tetrazolium bromide (MTT assay reagent), live/dead cell double staining kit, and DNA fragmentation imaging kit (TUNEL) were purchased from SigmaAldrich. Penicillin/streptomycin (10 $000 \mathrm{U} / \mathrm{mL})$, trypsin-EDTA (0.25\%), phosphate buffered silane (PBS), fetal bovine serum (FBS), JC-1 assay, $\mathrm{H}_{2}$ DCFDA assay, HMOX1 antibody, and goat antimouse $\operatorname{IgG}(\mathrm{H}+\mathrm{L})$ secondary antibody Alexa Fluor 488 conjugate were purchased from Thermo Fisher Scientific. mPEG-silane (MW 2k) was purchased from Creative PEGWorks. The FITC Annexin V Apoptosis Detection Kit with PI was purchased from BioLegend. VECTASHIELD antifade mounting medium with DAPI was purchased from VECTOR Laboratories.

\section{Synthesis of MSN@MON.}

A $20 \mathrm{~mL}$ aliquot of a CTAC $(2 \mathrm{~g})$ and TEA $(0.1 \mathrm{~g})$ aqueous solution was stirred for $30 \mathrm{~min}$. After transferring the system to an $80^{\circ} \mathrm{C}$ oil bath, $1 \mathrm{~mL}$ of TEOS was added and reacted for $1 \mathrm{~h}$. Next, $1 \mathrm{~mL}$ of an equal volume mixture of BTES and BETB was added and reacted for $4 \mathrm{~h}$. The products were centrifuged and washed with ethanol for several times. The CTAC residual was extracted with methanol of $\mathrm{NaCl}$.

\section{Synthesis of HMON.}

A $5 \mathrm{~mL}$ aliquot of the above as-synthesized MSN@MON aqueous solution was added to 25 $\mathrm{mL}$ of ultrapure water. After addition of $3 \mathrm{~mL}$ of ammonium hydroxide (w/v 28\%), the system was transferred to a $95^{\circ} \mathrm{C}$ oil bath and reacted under a nitrogen atmosphere for $3 \mathrm{~h}$. The resultant HMONs were centrifuged and washed with water for several times.

\section{Synthesis of HMON-PEG.}

The above as-synthesized HMON and $50 \mathrm{mg}$ of PEG-silane $(\mathrm{Mw}=2000)$ were added to 100 $\mathrm{mL}$ of ethanol via vigorous stirring. The system was transferred to a $78{ }^{\circ} \mathrm{C}$ oil bath and refluxed overnight. Finally, the resulting HMON-PEG products were centrifuged and washed with ethanol/water.

\section{Synthesis of HMOPMs.}

First, to modify thiol groups onto the surface of HMON-PEG, $150 \mu \mathrm{L}$ of MPTES, $200 \mu \mathrm{L}$ of $\mathrm{NH}_{3} \cdot \mathrm{H}_{2} \mathrm{O}$, and $40 \mathrm{mg}$ of $\mathrm{HMON}-\mathrm{PEG}$ were mixed in $30 \mathrm{~mL}$ of ethanol via vigorous stirring. After $10 \mathrm{~h}$ of reaction, the resulting HMON-PEG/-SH nanoparticles were collected and washed with ethanol by centrifugation. Second, POM was stably anchored onto HMONPEG surfaces based on metal-thiol coordination chemistry. The as-prepared HMON$\mathrm{PEG} / \mathrm{SH}$ and POM were mixed in $10 \mathrm{~mL}$ of ultrapure water with supersonic treatment for 15 
min. Finally, the resulting POM-attached HMON-PEG (HMOPMs) products were collected and resuspended in ultrapure water.

\section{Synthesis of HMOPMs-CO.}

The $\mathrm{Mn}_{2}(\mathrm{CO})_{10}$-loaded HMOPMs (denoted as HMOPMs-CO) were synthesized through three steps. First, to load $\mathrm{Mn}_{2}(\mathrm{CO})_{10}$ in HMON-PEG, $40 \mathrm{mg}$ of HMON-PEG and $100 \mathrm{mg}$ of $\mathrm{Mn}_{2}(\mathrm{CO})_{10}$ were mixed in $30 \mathrm{~mL}$ of ethanol for $12 \mathrm{~h}$ of vigorous stirring. Then the resulting $\mathrm{Mn}_{2}(\mathrm{CO})_{10} @ \mathrm{HMON}-\mathrm{PEG}$ products were centrifuged and washed with ethanol. Second, 150 $\mu \mathrm{L}$ of MPTES, $200 \mu \mathrm{L}$ of $\mathrm{NH}_{3} \cdot \mathrm{H}_{2} \mathrm{O}$, and the as-prepared $\mathrm{Mn}_{2}(\mathrm{CO})_{10} @ \mathrm{HMON}-\mathrm{PEG}$ were mixed in $30 \mathrm{~mL}$ of ethanol via vigorous stirring. After $10 \mathrm{~h}$ of reaction, the resulting $\mathrm{Mn}_{2}(\mathrm{CO})_{10} @ \mathrm{HMON}-\mathrm{PEG} / \mathrm{SH}$ nanoparticles were centrifuged and washed with ethanol. Third, the as-prepared $\mathrm{Mn}_{2}(\mathrm{CO})_{10} @ \mathrm{HMON}-\mathrm{PEG} / \mathrm{SH}$ and POM were mixed in $10 \mathrm{~mL}$ of ultrapure water with supersonic treatment for $15 \mathrm{~min}$. Then the resulting POM-attached $\mathrm{Mn}_{2}(\mathrm{CO})_{10} @ \mathrm{HMON}-\mathrm{PEG} / \mathrm{SH}$ (abbreviated as HMOPMs-CO) products were collected and dispersed in ultrapure water.

\section{CO Generation.}

A COP-1 probe (Ex/Em: $475 / 510 \mathrm{~nm}$ ) was used as a CO indicator. A $1 \mathrm{~mL}$ aliquot of HMOPMs-CO solution (preincubated with $10 \mathrm{mM} \mathrm{GSH}$ at $1 \mathrm{mg} \mathrm{Mo} / \mathrm{mL}$ for $24 \mathrm{~h}$ ) and the COP-1 probe working solution (final concentration, $1 \mu \mathrm{g} / \mathrm{mL}$ ) were mixed in an open-capped cuvette. The solution was exposed to an $808 \mathrm{~nm}$ laser at different fluences of $0,0.3,0.5$, and $1.0 \mathrm{~W} / \mathrm{cm}^{2}$ for $0.5 \mathrm{~h}$. The fluorescence spectra of the solution were monitored at selected time points $(0,1,2,3,4,5,6,7,8,9,10,12,15,20,25$, and $30 \mathrm{~min})$ under $475 \mathrm{~nm}$ excitation on a Hitachi F-7000 fluorescence spectrophotometer. The $\mathrm{CO}$ generation was determined by the fluorescence emission intensity at $510 \mathrm{~nm}$. To monitor in vitro $\mathrm{CO}$ release, cells were incubated with the COP-1 probe at a final concentration of $1 \mu \mathrm{g} / \mathrm{mL}$ for $0.5 \mathrm{~h}$. After incubating with HMOPM-CO solutions (GSH pretreated) at a concentration of $100 \mu \mathrm{g} \mathrm{Mo} / \mathrm{mL}$, the cells were exposed to an $808 \mathrm{~nm}$ irradiation at $0.3 \mathrm{~W} / \mathrm{cm}^{2}$ for $20 \mathrm{~min}$ or at $0.3,0.5$, and $1.0 \mathrm{~W} / \mathrm{cm}^{2}$ for $3 \mathrm{~min}$. Cells that received no irradiation were set as a control group. For confocal microscopy observation, the U87MG cells were washed with PBS, fixed with Z-fix solution, mounted with mounting medium with DAPI, and examined under confocal microscopy. For flow cytometry analysis, the cells were washed, trypsinized, collected, and finally analyzed.

\section{In Vitro Biological Effect with the CO Release.}

U87MG cells were incubated with $100 \mu \mathrm{g} \mathrm{Mo} / \mathrm{mL}$ HMOPM-CO solution (preincubated with $10 \mathrm{mM} \mathrm{GSH}$ at $1 \mathrm{mg} \mathrm{Mo} / \mathrm{mL}$ for $24 \mathrm{~h}$ ) and then exposed to an $808 \mathrm{~nm}$ irradiation at 0.3 $\mathrm{W} / \mathrm{cm}^{2}$ for $20 \mathrm{~min}$. In control groups, cells were treated with the same concentration of the HMOPMs-CO, an $808 \mathrm{~nm}$ irradiation, and PBS, respectively. After $24 \mathrm{~h}$ of the treatment, mitochondria membrane potential was tested with JC-1 assay (Invitrogen, M34152) and the intracellular ROS level was measured with $\mathrm{H}_{2}$ DCFDA assay (Invitrogen, D399) through both confocal microscopy and flow cytometry. 


\section{In Vivo PET Imaging.}

The ${ }^{64} \mathrm{Cu}$-labeled HMOPMs or HMONs were systematically administrated into U87MG tumor-bearing mice $(n=4)$ at $100 \mu \mathrm{Ci}$. The PET images were performed on a micro Inveon PET scanner at 1, 4, 24, and $48 \mathrm{~h}$ p.i. Regions of interest (ROIs) were circled, and the corresponding radioactivities were quantified on the tumors, livers, and spleens in the decaycorrected whole-body coronal images. After the imaging, the mice were scarified, and major organs were collected. The organs were weighted, and their radioactivities were measured by gamma countering.

\section{In Vivo Safety Evaluation.}

The evaluation was performed on Balb/c mice. HMOPM-CO or HMOPM was intravenously injected into mice at a dosage of $10 \mathrm{mg} \mathrm{Mo} / \mathrm{kg}(n=5)$. PBS was administrated as a control $(n=5)$. The mice body weight was monitored every other day. After 7 days, mice were sacrificed. Blood was withdrawn for whole blood count and blood biochemical index analysis. Major organs (i.e., heart, kidneys, lung, spleen, and liver) were collected for H\&E staining analysis.

\section{Synergistic PTT/Gas Therapy in Vivo.}

When the tumor size reached around $100 \mathrm{~mm}^{3}$, HMOPMs-CO $(10 \mathrm{mg} \mathrm{Mo} / \mathrm{kg})$ were intravenously injected into the tumor-bearing mice $(n=5)$. After $24 \mathrm{~h}$, tumors were exposed to an $808 \mathrm{~nm}$ irradiation $\left(1.0 \mathrm{~W} / \mathrm{cm}^{2}, 5 \mathrm{~min}\right)$. The six control groups ( $n=5 /$ group) were also investigated: (1) HMOPMs + irradiation $\left(1.0 \mathrm{~W} / \mathrm{cm}^{2}, 5 \mathrm{~min}\right)$; (2) HMOPMs-CO + irradiation ( $\left.0.3 \mathrm{~W} / \mathrm{cm}^{2}, 20 \mathrm{~min}\right)$; (3) HMOPMs + irradiation $\left(0.3 \mathrm{~W} / \mathrm{cm}^{2}, 20 \mathrm{~min}\right)$; (4) HMOPMs-CO only; (5) PBS and + irradiation $\left(1.0 \mathrm{~W} / \mathrm{cm}^{2}, 5 \mathrm{~min}\right)$; and (6) PBS only. Tumor sizes $\left(=\right.$ length $\times$ width $\left.^{2} / 2\right)$ and mice body weights were monitored every other day. In addition, tumors were collected after $24 \mathrm{~h}$ of the treatments and major organs were harvested at the end of the therapy for histological analysis.

The acidic TME will drive the self-assembly of HMOPM-CO via intermolecular hydrogen bonding of POM, leading to enhanced tumor retention and accumulation. Meanwhile, the reductive TME will activate $\mathrm{Mo}(\mathrm{VI})$-to-Mo(V) conversion within $\mathrm{POM}$, endowing the HMOPM-CO with outstanding PA contrast and photothermal effect, which will further trigger the decomposition of the $\mathrm{Mn}_{2}(\mathrm{CO})_{10}$ payload for $\mathrm{CO}$ release. This intelligent acidity/ reducibility dual-responsive nanoplatform is expected to achieve enhanced tumor accumulation via in situ self-assembly and precise tumor-specific PTT/CO gas synergistic therapy.

\section{Supplementary Material}

Refer to Web version on PubMed Central for supplementary material.

\section{ACKNOWLEDGMENTS}

We gratefully acknowledge Li Yao from Howard Hughes Medical Institute for the help with the TOC figure and schematic illustration of nanoparticle synthesis and self-assembly. This work was supported by the Intramural Research Program of the National Institute of Biomedical Imaging and Bioengineering, National Institutes of Health. We thank the National Institutes of Health (R01EB022596, J.X., and R01NS093314, J.X.) and the National 
Science Foundation (NSF1552617, J.X.) for their funding support. We are also thankful for the support from the National Natural Science Foundation of China (51602203, W.F.) and the Youth Innovation Promotion Association of Chinese Academy of Sciences (2016269, Z.S.).

\section{REFERENCES}

(1). Zhao D; Feng J; Huo Q; Melosh N; Fredrickson GH; Chmelka BF; Stucky GD Triblock Copolymer Syntheses of Mesoporous Silica with Periodic 50 to 300 Angstrom Pores. Science 1998, 279, 548-552. [PubMed: 9438845]

(2). Davis ME Ordered Porous Materials for Emerging Applications. Nature 2002, 417, 813-821. [PubMed: 12075343]

(3). Li Y; Shi J Hollow-Structured Mesoporous Materials: Chemical Synthesis, Functionalization and Applications. Adv. Mater 2014, 26, 3176-3205. [PubMed: 24687906]

(4). Tang F; Li L; Chen D Mesoporous Silica Nanoparticles: Synthesis, Biocompatibility and Drug Delivery. Adv. Mater 2012, 24, 1504-1534. [PubMed: 22378538]

(5). Wen J; Yang K; Liu F; Li H; Xu Y; Sun S Diverse Gatekeepers for Mesoporous Silica Nanoparticle Based Drug Delivery Systems. Chem. Soc. Rev 2017, 46, 6024-6045. [PubMed: 28848978]

(6). Chen Y; Chen H; Shi J Construction of Homogenous/ Heterogeneous Hollow Mesoporous Silica Nanostructures by Silica-Etching Chemistry: Principles, Synthesis, and Applications. Acc. Chem. Res 2014, 47, 125-137. [PubMed: 23944328]

(7). Luo Z; Ding X; Hu Y; Wu S; Xiang Y; Zeng Y; Zhang B; Yan H; Zhang H; Zhu L; Liu J; Li J; Cai K; Zhao Y Engineering A Hollow Nanocontainer Platform with Multifunctional Molecular Machines for Tumor-Targeted Therapy in Vitro and in Vivo. ACS Nano 2013, 7, 10271-10284. [PubMed: 24127723]

(8). Croissant JG; Fatieiev Y; Khashab NM Degradability and Clearance of Silicon, Organosilica, Silsesquioxane, Silica Mixed Oxide, and Mesoporous Silica Nanoparticles. Adv. Mater 2017, 29, 1604634.

(9). Chen Y; Shi J Chemistry of Mesoporous Organosilica in Nanotechnology: Molecularly OrganicInorganic Hybridization into Frameworks. Adv. Mater 2016, 28, 3235-3272. [PubMed: 26936391]

(10). Teng Z; Wang C; Tang Y; Li W; Bao L; Zhang X; Su X; Zhang F; Zhang J; Wang S; Zhao D; Lu G Deformable Hollow Periodic Mesoporous Organosilica Nanocapsules for Significantly Improved Cellular Uptake. J. Am. Chem. Soc 2018, 140, 1385-1393. [PubMed: 29281272]

(11). Teng Z; Su X; Zheng Y; Zhang J; Liu Y; Wang S; Wu J; Chen G; Wang J; Zhao D; Lu G A Facile Multi-interface Transformation Approach to Monodisperse Multiple-Shelled Periodic Mesoporous Organosilica Hollow Spheres. J. Am. Chem. Soc 2015, 137, 7935-7944. [PubMed: 26030506]

(12). Zhou M; Du X; Li W; Li X; Huang H; Liao Q; Shi B; Zhang X; Zhang M One-Pot Synthesis of Redox-Triggered Biodegradable Hybrid Nanocapsules with A Disulfide-Bridged Silsesquioxane Framework for Promising Drug Delivery. J. Mater. Chem. B 2017, 5, 4455-4469.

(13). Fan W; Lu N; Huang P; Liu Y; Yang Z; Wang S; Yu G; Liu Y; Hu J; He Q; Qu J; Wang T; Chen $X$ Glucose-Responsive Sequential Generation of Hydrogen Peroxide and Nitric Oxide for Synergistic Cancer Starving-Like/Gas Therapy. Angew. Chem., Int. Ed 2017, 56, 1229-1233.

(14). Chen Y; Xu P; Chen H; Li Y; Bu W; Shu Z; Li Y; Zhang J; Zhang L; Pan L; Cui X; Hua Z; Wang J; Zhang L; Shi J Colloidal HPMO Nanoparticles: Silica-Etching Chemistry Tailoring, Topological Transformation, and Nano-Biomedical Applications. Adv. Mater 2013, 25, 31003105. [PubMed: 23418108]

(15). Bertrand N; Wu J; Xu X; Kamaly N; Farokhzad OC Cancer Nanotechnology: The Impact of Passive and Active Targeting in the Era of Modern Cancer Biology. Adv. Drug Delivery Rev 2014, 66, 2-25.

(16). Stewart MP; Sharei A; Ding X; Sahay G; Langer R; Jensen KF In Vitro and ex Vivo Strategies for Intracellular Delivery. Nature 2016, 538, 183-192. [PubMed: 27734871]

(17). Tsvetkova Y; Beztsinna N; Baues M; Klein D; Rix A; Golombek SK; Rawashdeh WA; Gremse F; Barz M; Koynov K; Banala S; Lederle W; Lammers T; Kiessling F Balancing Passive and Active 
Targeting to Different Tumor Compartments Using Riboflavin-Functionalized Polymeric Nanocarriers. Nano Lett 2017, 17, 4665-4674. [PubMed: 28715227]

(18). Polo E; Collado M; Pelaz B; del Pino P Advances Toward More Efficient Targeted Delivery of Nanoparticles in Vivo: Under-standing Interactions Between Nanoparticles and Cells. ACS Nano 2017, 11, 2397-2402. [PubMed: 28267316]

(19). Pan L; Liu J; He Q; Shi J MSN-Mediated Sequential Vascular-to-Cell Nuclear-Targeted Drug Delivery for Efficient Tumor Regression. Adv. Mater 2014, 26, 6742-6748. [PubMed: 25159109]

(20). Idris NM; Gnanasammandhan MK; Zhang J; Ho PC; Mahendran R; Zhang Y In Vivo Photodynamic Therapy Using Upconversion Nanoparticles as Remote-Controlled Nanotransducers. Nat. Med 2012, 18, 1580-1585. [PubMed: 22983397]

(21). Shen Z; Chen T; Ma X; Ren W; Zhou Z; Zhu G; Zhang A; Liu Y; Song J; Li Z; Ruan H; Fan W; Lin L; Munasinghe J; Chen X; Wu A Multifunctional Theranostic Nanoparticles Based on Exceedingly Small Magnetic Iron Oxide Nanoparticles for T1-Weighted Magnetic Resonance Imaging and Chemotherapy. ACS Nano 2017, 11, 10992-11004. [PubMed: 29039917]

(22). Sun B; Feng S Trastuzumab-Functionalized Nanoparticles of Biodegradable Copolymers for Targeted Delivery of Docetaxel. Nanomedicine 2009, 4, 431-445. [PubMed: 19505246]

(23). Zhen Z; Tang W; Chen H; Lin X; Todd T; Wang G; Cowger T; Chen X; Xie J RGD-Modified Apoferritin Nanoparticles for Efficient Drug Delivery to Tumors. ACS Nano 2013, 7, 4830-4837. [PubMed: 23718215]

(24). Chen H; Zhang W; Zhu G; Xie J; Chen X Rethinking Cancer Nanotheranostics. Nat. Rev. Mater 2017, 2, 17024. [PubMed: 29075517]

(25). Prabhakar U; Maeda H; Jain RK; Sevick-Muraca EM; Zamboni W; Farokhzad OC; Barry ST; Gabizon A; Grodzinski P; Blakey DC Challenges and Key Considerations of the Enhanced Permeability and Retention Effect for Nanomedicine Drug Delivery in Oncology. Cancer Res 2013, 73, 2412-2417. [PubMed: 23423979]

(26). Perry JL; Reuter KG; Luft JC; Pecot CV; Zamboni W; DeSimone JM Mediating Passive Tumor Accumulation through Particle Size, Tumor Type, and Location. Nano Lett 2017, 17, 2879-2886. [PubMed: 28287740]

(27). Sykes EA; Chen J; Zheng G; Chan WCW Investigating the Impact of Nanoparticle Size on Active and Passive Tumor Targeting Efficiency. ACS Nano 2014, 8, 5696-5706. [PubMed: 24821383]

(28). Yang Z; Tian R; Wu J; Fan Q; Yung BC; Niu G; Jacobson O; Wang Z; Liu G; Yu G; Huang W; Song J; Chen X Impact of Semiconducting Perylene Diimide Nanoparticle Size on Lymph Node Mapping and Cancer Imaging. ACS Nano 2017, 11, 4247-4255. [PubMed: 28345873]

(29). Huang P; Chen Y; Lin H; Yu L; Zhang L; Wang L; Zhu Y; Shi J Molecularly Organic/Inorganic Hybrid Hollow Mesoporous Organosilica Nanocapsules with Tumor-Specific Biodegradability and Enhanced Chemotherapeutic Functionality. Biomaterials 2017, 125, 23-37. [PubMed: 28226244]

(30). Huang P; Gao Y; Lin J; Hu H; Liao HS; Yan X; Tang Y; Jin A; Song J; Niu G; Zhang G; Horkay F; Chen X Tumor-Specific Formation of Enzyme-Instructed Supramolecular Self-Assemblies as Cancer Theranostics. ACS Nano 2015, 9, 9517-9527. [PubMed: 26301492]

(31). Zhang C; Bu W; Ni D; Zuo C; Cheng C; Li Q; Zhang L; Wang Z; Shi J A Polyoxometalate Cluster Paradigm with Self-Adaptive Electronic Structure for Acidity/Reducibility-Specific Photo-thermal Conversion. J. Am. Chem. Soc 2016, 138, 8156-8164. [PubMed: 27264421]

(32). Chen Y; Meng Q; Wu M; Wang S; Xu P; Chen H; Li Y; Zhang L; Wang L; Shi J Hollow Mesoporous Organosilica Nanoparticles: a Generic Intelligent Framework-Hybridization Approach for Biomedicine. J. Am. Chem. Soc 2014, 136, 16326-16334. [PubMed: 25343459]

(33). Lu Z; Gao C; Zhang Q; Chi M; Howe JY; Yin Y Direct Assembly of Hydrophobic Nanoparticles to Multifunctional Structures. Nano Lett 2011, 11, 3404-3412. [PubMed: 21732588]

(34). Wang Y; Raula M; Wang Y; Zeiri O; Chakraborty S; Gan-Or G; Gadot E; Weinstock IA Polyoxometalate-Engineered Building Blocks with Gold Cores for the Self-Assembly of Responsive Water-Soluble Nanostructures. Angew. Chem., Int. Ed 2017, 56, 7083-7087. 
(35). Slowing II; Wu CW; Vivero-Escoto JL; Lin VS Mesoporous Silica Nanoparticles for Reducing Hemolytic Activity towards Mammalian Red Blood Cells. Small 2009, 5, 57-62. [PubMed: 19051185]

(36). Lin YS; Haynes CL Impacts of Mesoporous Silica Nanoparticle Size, Pore Ordering, and Pore Integrity on Hemolytic Activity. J. Am. Chem. Soc 2010, 132, 4834-4842. [PubMed: 20230032]

(37). Liu T; Diemann E; Li H; Dress AW; Muller A Self-Assembly in Aqueous Solution of WheelShaped Mo154 Oxide Clusters into Vesicles. Nature 2003, 426, 59-62. [PubMed: 14603315]

(38). Ni D; Jiang D; Valdovinos HF; Ehlerding EB; Yu B; Barnhart TE; Huang P; Cai W Bioresponsive Polyoxometalate Cluster for Redox-Activated Photoacoustic Imaging-Guided Photo-thermal Cancer Therapy. Nano Lett 2017, 17, 3282-3289. [PubMed: 28418679]

(39). Geisow MJ; Evans WH pH in the Endosome. Measurements During Pinocytosis and ReceptorMediated Endocytosis. Exp. Cell Res 1984, 150, 36-46. [PubMed: 6198190]

(40). Murphy RF; Powers S; Cantor CR Endosome pH Measured in Single Cells by Dual Fluorescence Flow Cytometry: Rapid Acidification of Insulin to pH 6. J. Cell Biol 1984, 98, 1757-1762. [PubMed: 6144684]

(41). Li WP; Su CH; Tsao LC; Chang CT; Hsu YP; Yeh CS Controllable CO Release Following NearInfrared Light-Induced Cleavage of Iron Carbonyl Derivatized Prussian Blue Nanoparticles for CO-Assisted Synergistic Treatment. ACS Nano 2016, 10, 11027-11036. [PubMed: 28024357]

(42). Wu M; Meng Q; Chen Y; Du Y; Zhang L; Li Y; Zhang L; Shi J Large-Pore Ultrasmall Mesoporous Organosilica Nanoparticles: Micelle/Precursor Co-templating Assembly and Nuclear-Targeted Gene Delivery. Adv. Mater 2015, 27, 215-222. [PubMed: 25423915]

(43). Motterlini R; Otterbein LE The Therapeutic Potential of Carbon Monoxide. Nat. Rev. Drug Discovery 2010, 9, 728-743. [PubMed: 20811383]

(44). Wegiel B; Gallo D; Csizmadia E; Harris C; Belcher J; Vercellotti GM; Penacho N; Seth P; Sukhatme V; Ahmed A; Pandolfi PP; Helczynski L; Bjartell A; Persson JL; Otterbein LE Carbon Monoxide Expedites Metabolic Exhaustion to Inhibit Tumor Growth. Cancer Res 2013, 73, 7009-7021. [PubMed: 24121491]

(45). Zheng D; Li B; Li C; Xu L; Fan J; Lei Q; Zhang X Photocatalyzing CO2 to CO for Enhanced Cancer Therapy. Adv. Mater 2017, 29, 1703822.

(46). Liu Y; Zhang J; Zuo C; Zhang Z; Ni D; Zhang C; Wang J; Zhang H; Yao Z; Bu W Upconversion Nano-Photosensitizer Targeting into Mitochondria for Cancer Apoptosis Induction and Cyt C Fluorescence Monitoring. Nano Res 2016, 9, 3257-3266.

(47). Park H; Yang J; Lee J; Haam S; Choi IH; Yoo KH Multifunctional Nanoparticles for Combined Doxorubicin and Photo-thermal Treatments. ACS Nano 2009, 3, 2919-2926. [PubMed: 19772302]

(48). Lu N; Fan W; Yi X; Wang S; Wang Z; Tian R; Jacobson O; Liu Y; Yung BC; Zhang G; Teng Z; Yang K; Zhang M; Niu G; Lu G; Chen X Biodegradable Hollow Mesoporous Organosilica Nanotheranostics for Mild Hyperthermia-Induced Bubble-Enhanced Oxygen-Sensitized Radiotherapy. ACS Nano 2018, 12, 1580-1591. [PubMed: 29384652]

(49). Fan W; Yung BC; Huang P; Chen X Nanotechnology for Multimodal Synergistic Cancer Therapy. Chem. Rev 2017, 117, 13566-13638. [PubMed: 29048884]

(50). Tang W; Yang Z; Wang S; Wang Z; Song J; Yu G; Fan W; Dai Y; Wang J; Shan L; Niu G; Fan Q; Chen X Organic Semiconducting Photoacoustic Nanodroplets for Laser-Activatable Ultrasound Imaging and Combinational Cancer Therapy. ACS Nano 2018, 12, 2610-2622. [PubMed: 29451774]

(51). Srisook K; Han SS; Choi HS; Li MH; Ueda H; Kim C; Cha YN CO from Enhanced HO Activity or from CORM-2 Inhibits both $\mathrm{O}_{2}-$ and NO Production and Downregulates HO-1 Expression in LPS-Stimulated Macrophages. Biochem. Pharmacol 2006, 71, 307-318. [PubMed: 16329999] 


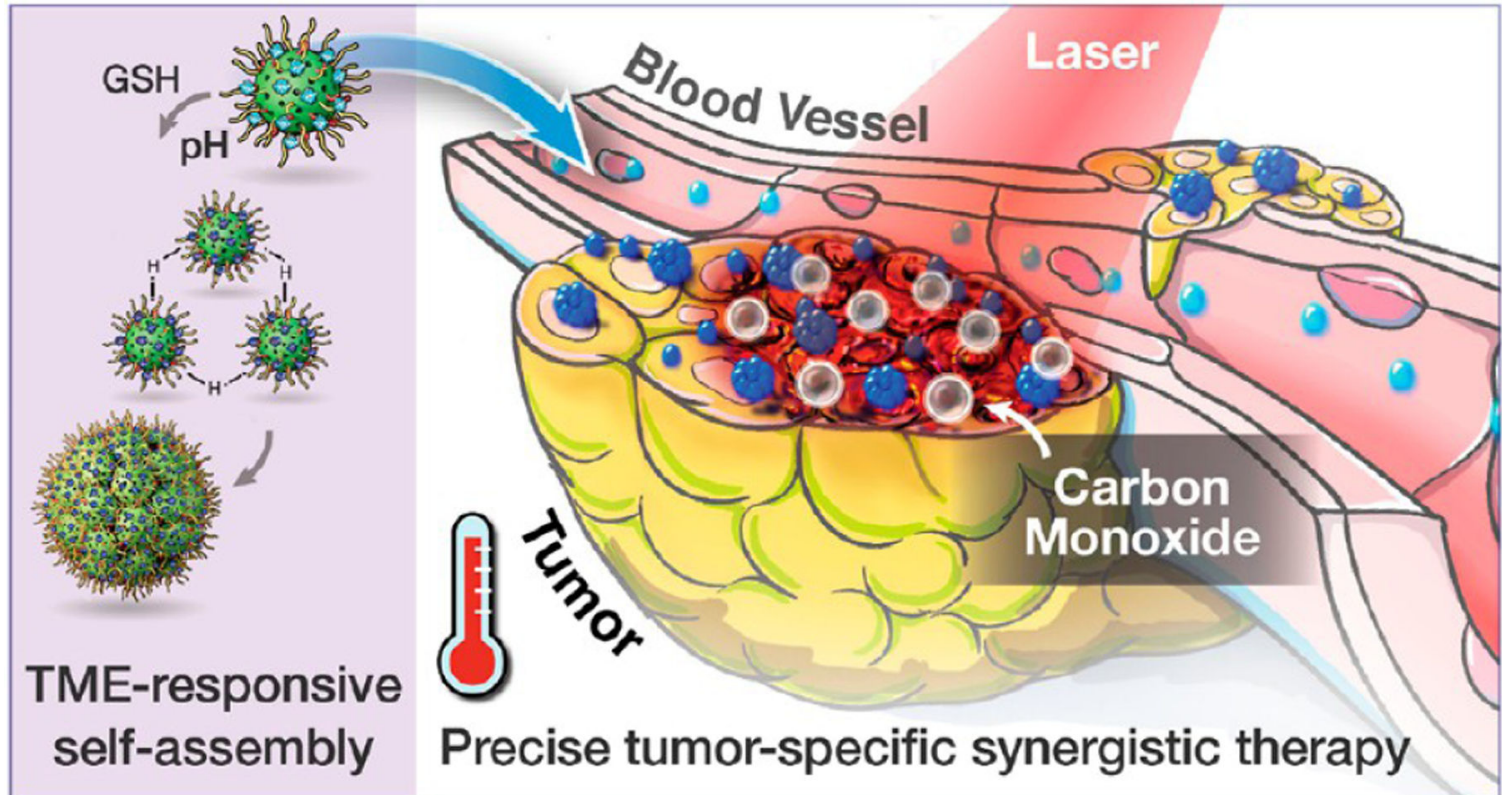

Scheme 1.

Schematic Illustration of the $\mathrm{Mn}_{2}(\mathrm{CO})_{10}$-Loaded and POM Surface-Modified Hollow Mesoporous Organosilica Nanoplatform, HMOPM-CO, for Tumor Microenvironment (TME)-Responsive Self-Assembly and Precise Synergistic Therapy 

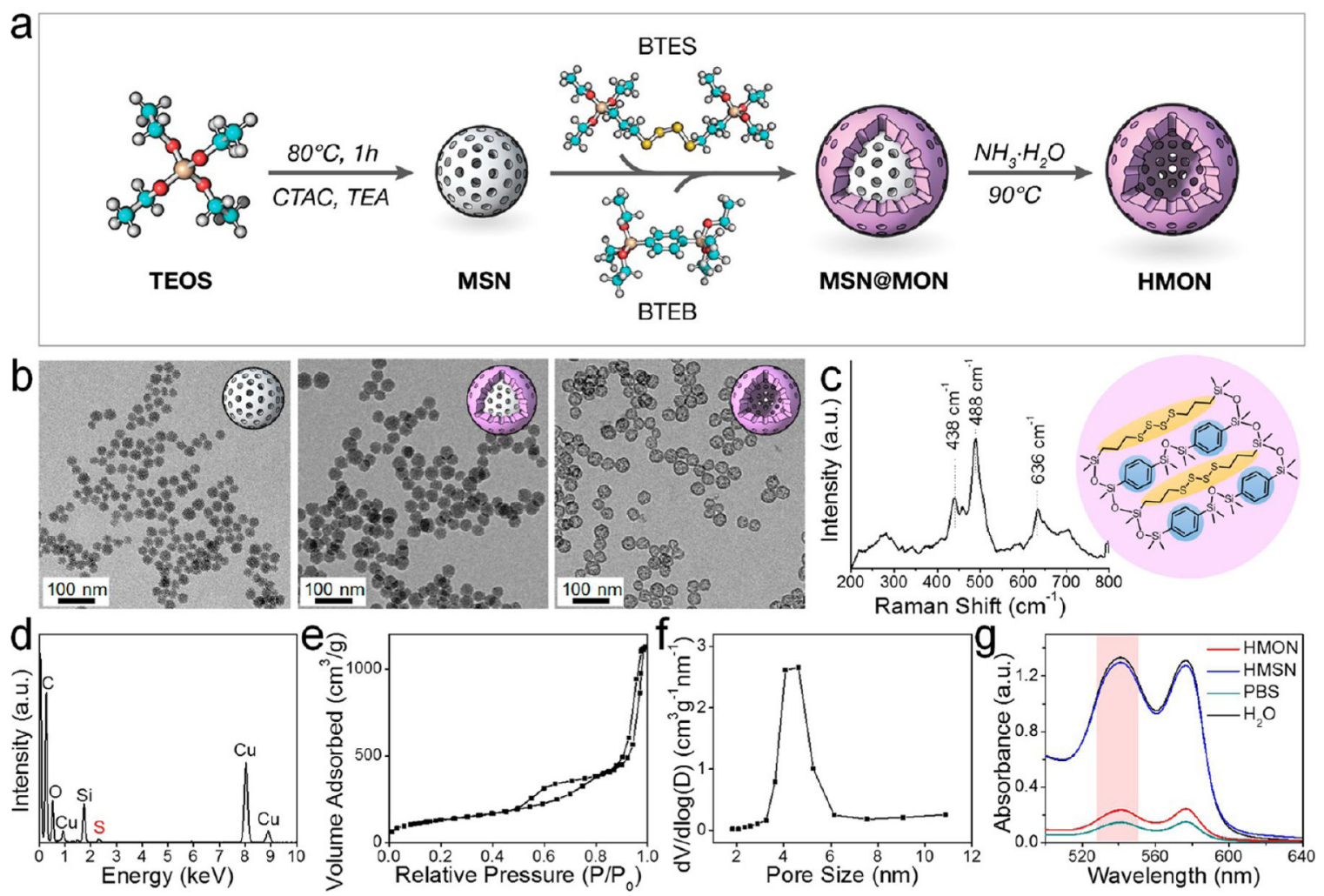

Figure 1.

Preparation and characterization of sub-50 nm thioether/phenylene dual-hybridized HMON. (a) Schematic synthesis route. MSN was first synthesized as a hard template. Through cohydrolysis of BTES and BTEB, core/shell structured MSN@MON was fabricated with thioether and phenylene moieties incorporated in the MON framework. The final HMON was fabricated via a special "ammonia-assisted hot water etching" strategy. (b) TEM images of MSN, MSN@MON, and HMON. Scale bar, $100 \mathrm{~nm}$. (c) Raman spectrum. The shift at $438 / 488$ and $636 \mathrm{~cm}^{-1}$ represented specific stretching vibrations of $-\mathrm{S}-\mathrm{S}-$ bond and $-\mathrm{S}-\mathrm{C}-$ bond, respectively. Insert: schematic illustration of the thioether/ phenylene dual-hybridized framework. (d) Energy-dispersive X-ray spectroscopy (EDS) spectrum. (e) $\mathrm{N}_{2}$ adsorption -desorption isotherm and (f) corresponding pore-size distribution, which showed a large surface area of $475.2 \mathrm{~m}^{2} / \mathrm{g}$ and an average pore size of around $4.3 \mathrm{~nm}$, respectively. (g) UV -vis spectra of supernatant after centrifugation of HMON or HMSN treated RBCs. Ultrapure water and PBS were set as the positive and negative control, respectively. The dual-hybridized HMON featured with significantly lower hemolysis relative to HMSN. 

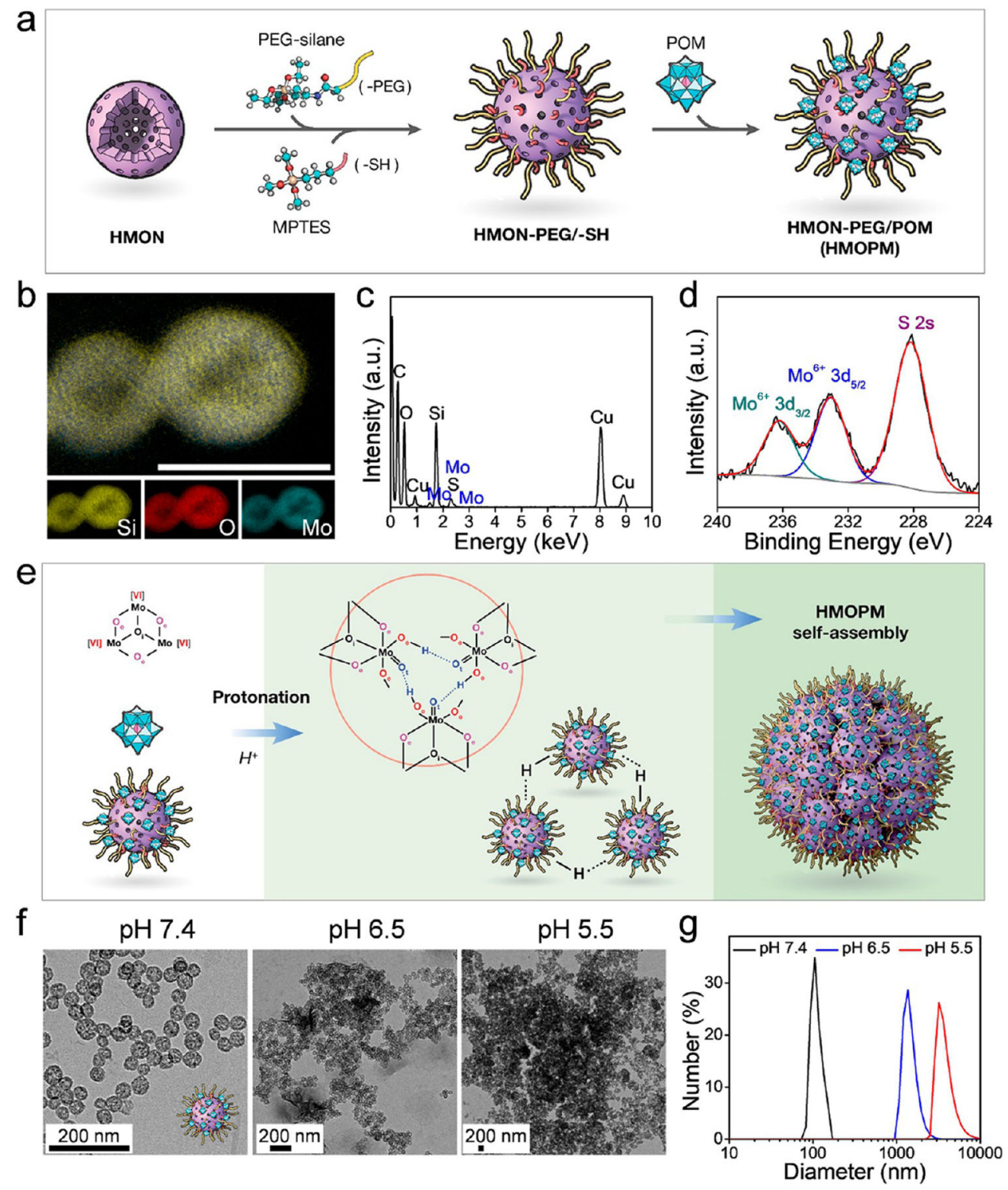

Figure 2.

Preparation and characterization of HMOPM. (a) Schematic synthesis route. The Mo-based polyoxometalate (POM) clusters were anchored onto the HMON surface through Mo-thiol coordination chemistry. (b) Elemental mapping. A hollow nanostructure of HMON with Mo homogeneously distributed on the shell was observed. Scale bar, $50 \mathrm{~nm}$. (c) EDS data validated the presence of Mo. (d) X-ray photoelectron spectroscopy (XPS) deconvoluted spectra for Mo 3d orbitals demonstrated that all the Mo element was consistent in one oxidation state of Mo (VI), indicating no unwanted redox chemistry reactions happened during the POM functionalization. (e) Schematic diagram proposed for the acidity-driven self-assembly. Hydrogen bonding was the major driving force for the assembly. (f) TEM images at different $\mathrm{pH}$ values. The uniformly distributed hollow structures $(\mathrm{pH}=7.4)$ would self-assemble to microsized clusters $(\mathrm{pH}=6.5)$ and even larger aggregates $(\mathrm{pH}=5.5)$ with successive acidifications. Scale bar, $200 \mathrm{~nm}$. (g) Dynamic light scattering (DLS) size 
measurement at different $\mathrm{pH}$ values corresponding to (f). The hydrodynamic size of HMOPM increased from $106.1 \pm 11.1 \mathrm{~nm}$ to $1367.5 \pm 272.7$ and $3568.4 \pm 891.8 \mathrm{~nm}$ with consecutive acidifications from $\mathrm{pH}=7.4$ to 6.5 and 5.5 . 


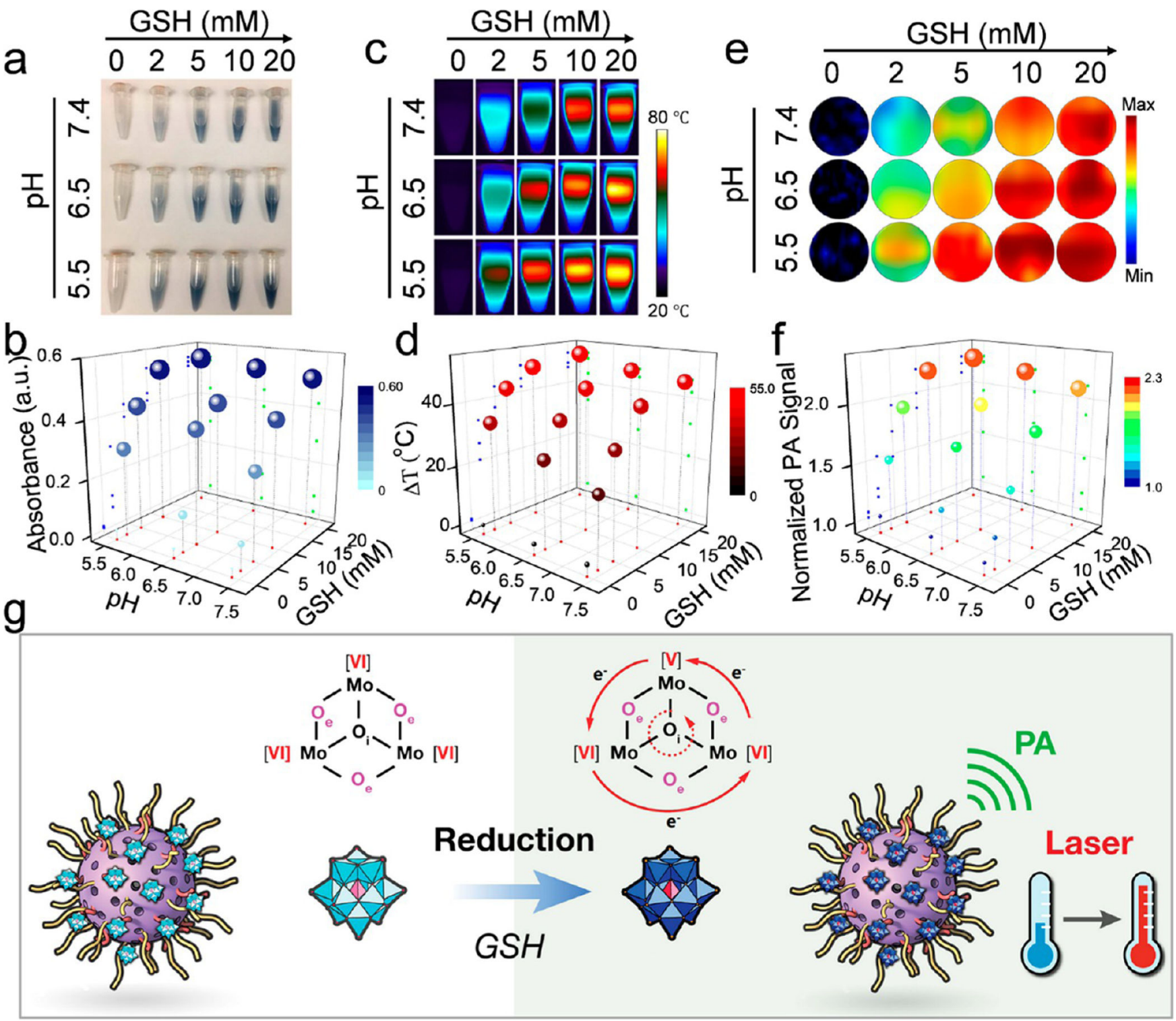

Figure 3.

Acidity and reducibility dual-responsive photothermal conversion and photoacoustic (PA) contrast of HMOPM. (a) Digital photographs, (b) UV-vis absorbance changes, (c) photothermal heating images, (d) temperature rises, (e) PA images, and (f) PA signal changes of HMOPM solutions under successive acidifications and reductions. An $808 \mathrm{~nm}$ irradiation at $1.0 \mathrm{~W} / \mathrm{cm}^{2}$ for $5 \mathrm{~min}$ was applied to evaluate the photothermal effect. The photothermal conversion and PA contrast could be activated at reductive environments. Stronger photothermal effect and higher PA contrast were achieved at higher acidity and, more significantly, at stronger reducibility. (g) Schematic illustration of the reducibilityactivatable photothermal and PA performance of HMOPM through the Mo(VI)-to-Mo(V) conversion within POM. 
a

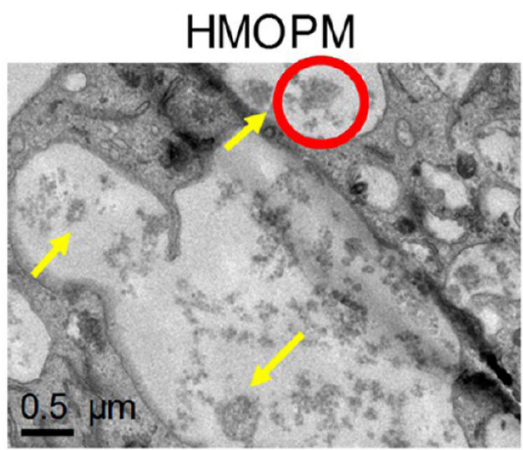

C

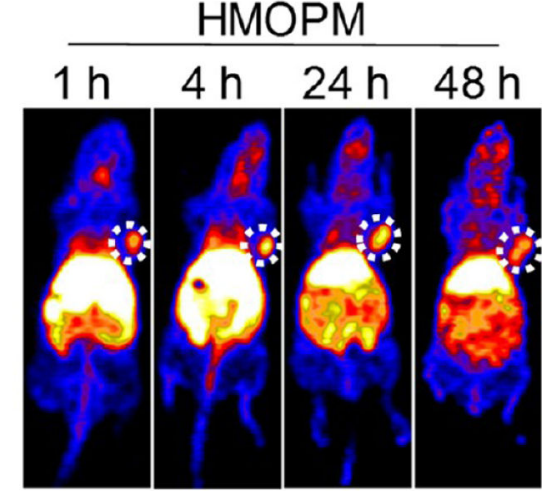

e

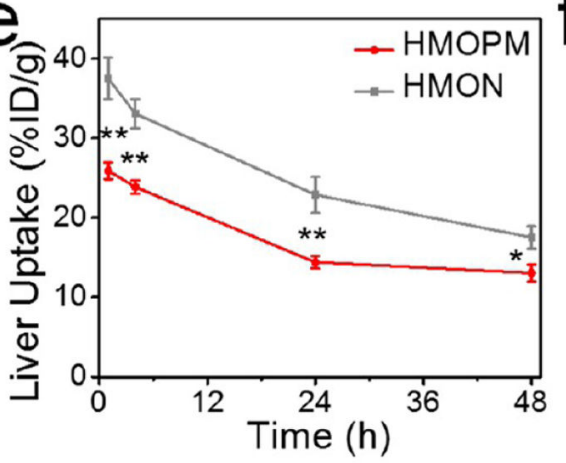

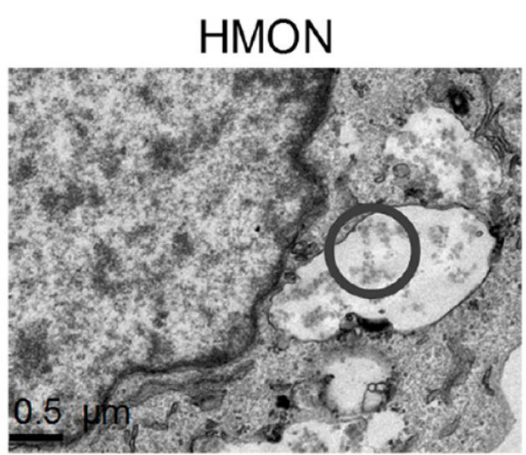

HMON
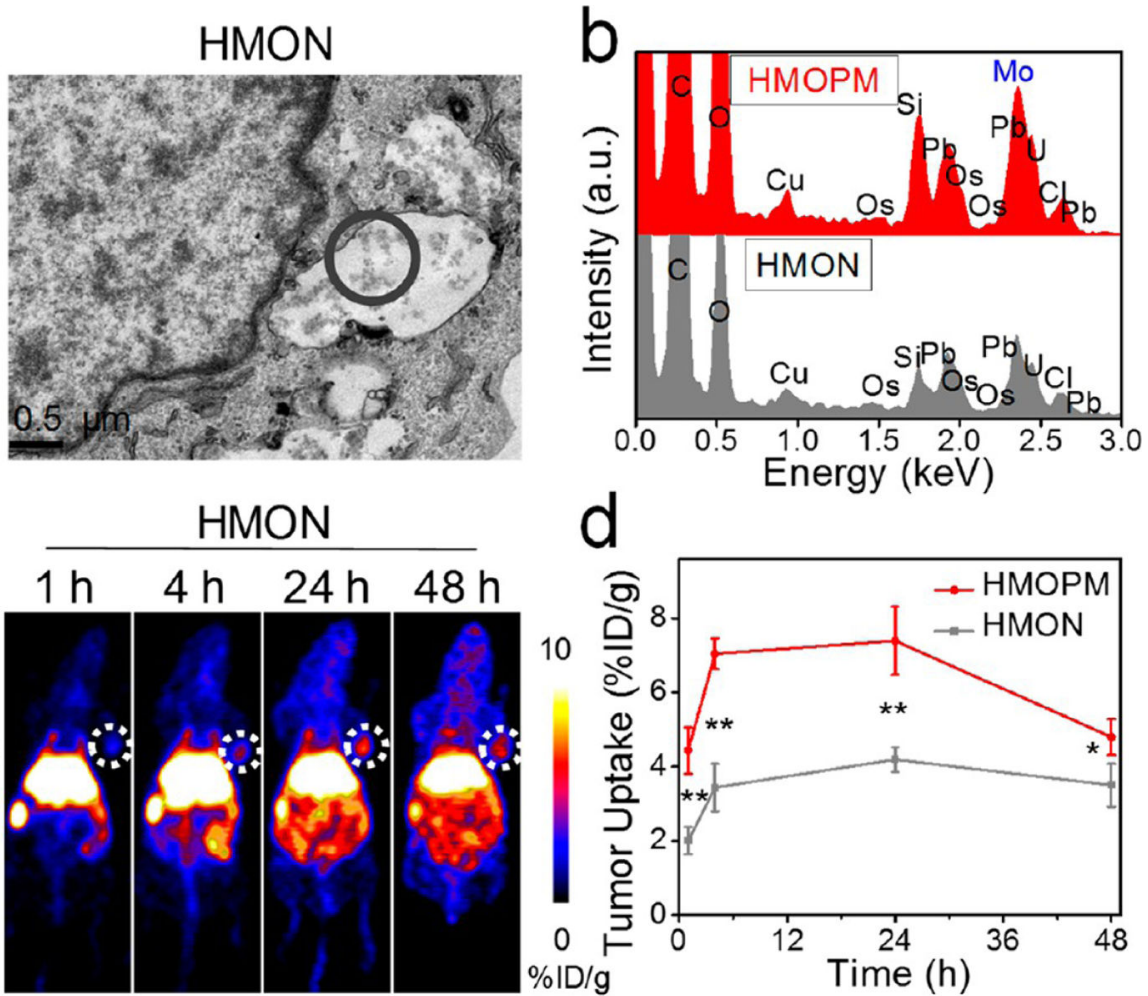

d
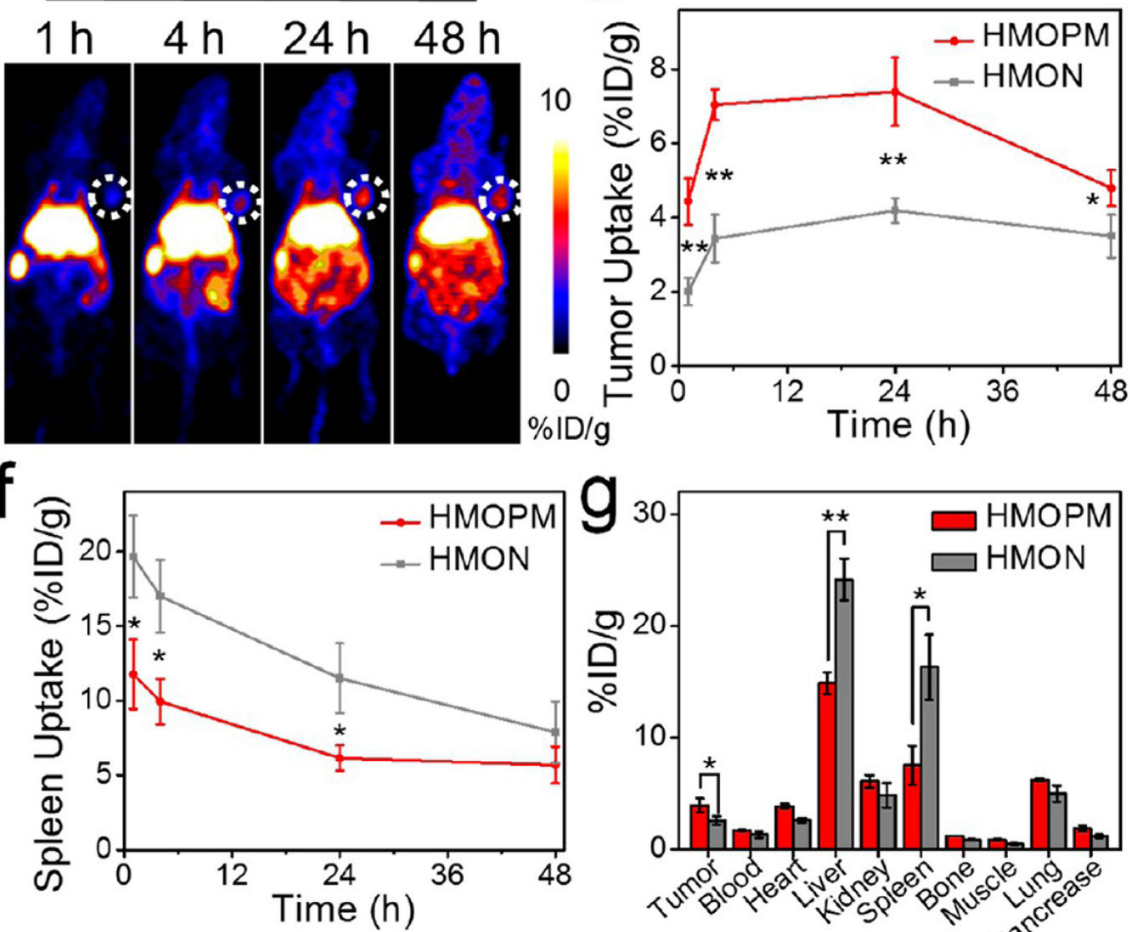

Figure 4.

Enhanced tumor accumulation based on the acidity-driven in situ self-assembly of HMOPM.

(a) Bio-TEM images of U87MG cells incubated with HMOPM or HMON. Scale bar, 0.5 $\mu \mathrm{m}$. Many HMOPM aggregates (marked with yellow arrows) were clearly observed in the endosomes at $\mathrm{pH}$ 5.0-6.0, while no apparent assemblies were found in the HMON-treated cells. (b) EDS elemental analysis of the correspondingly circled regions in (a). (c)

Representative PET images of U87MG tumor-bearing mice at 1, 4, 24, $48 \mathrm{~h}$ postinjection (p.i.) of the ${ }^{64} \mathrm{Cu}$-labeled HMOPM or HMON. Tumors were circled with white dots. (d-f) PET quantification on tumor (d), liver (e), and spleen (f) uptake ( $n=4$, mean \pm s.d.). (g) Biodistribution of the ${ }^{64} \mathrm{Cu}$-labeled HMOPM or HMON at 48 h p.i. $(n=4$, mean \pm s.d.). Significantly enhanced tumor accumulation and strikingly decreased liver and spleen uptake were observed in the HMOPM-treated mice comparing to the HMON-treated ones. $* P<$ 0.05 . ** $P<0.01$. 

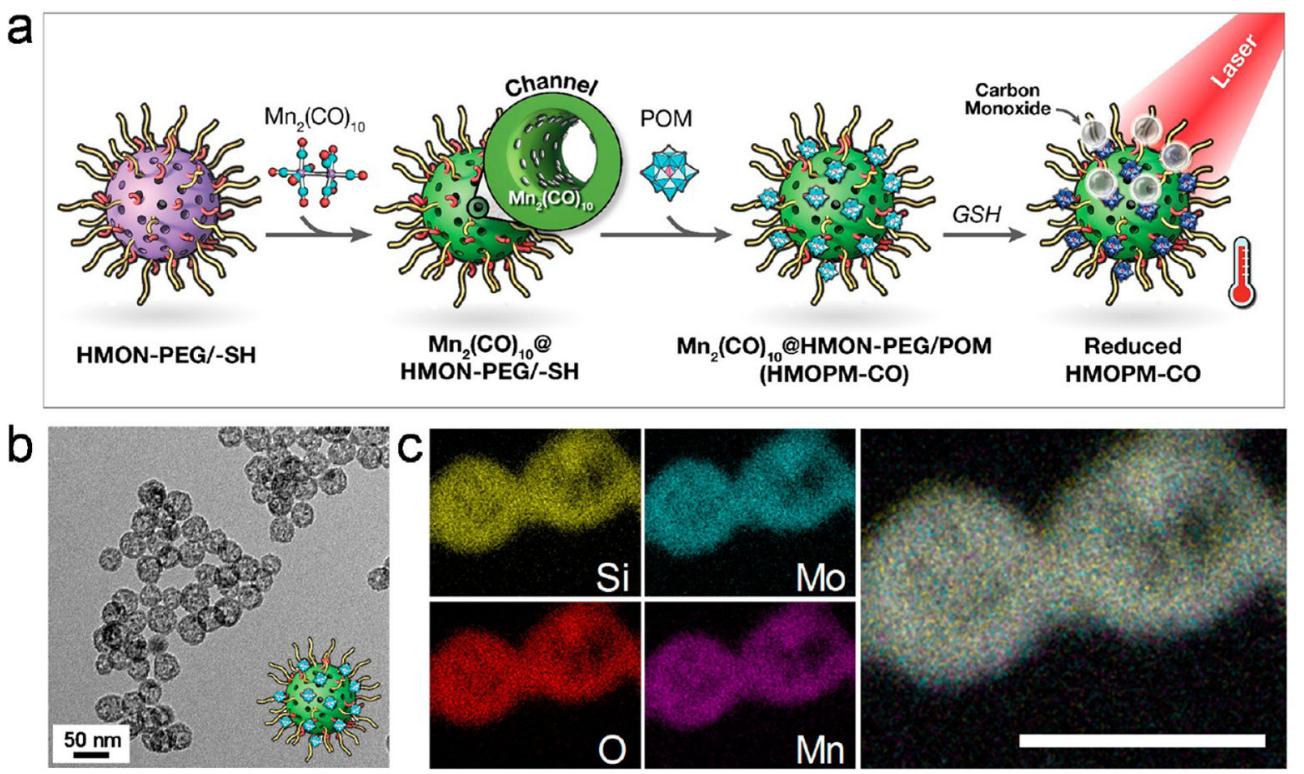

d
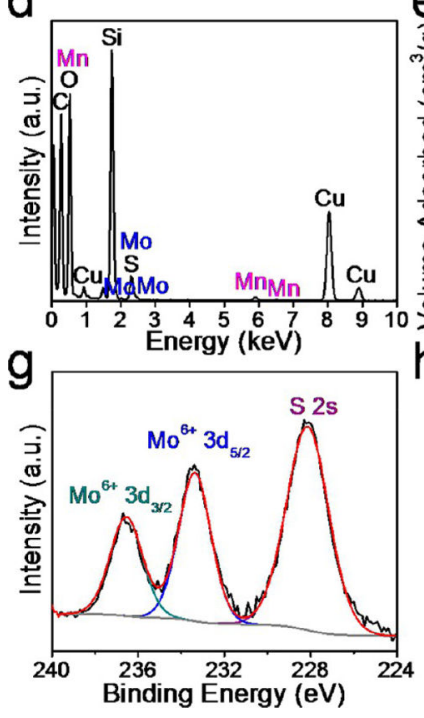
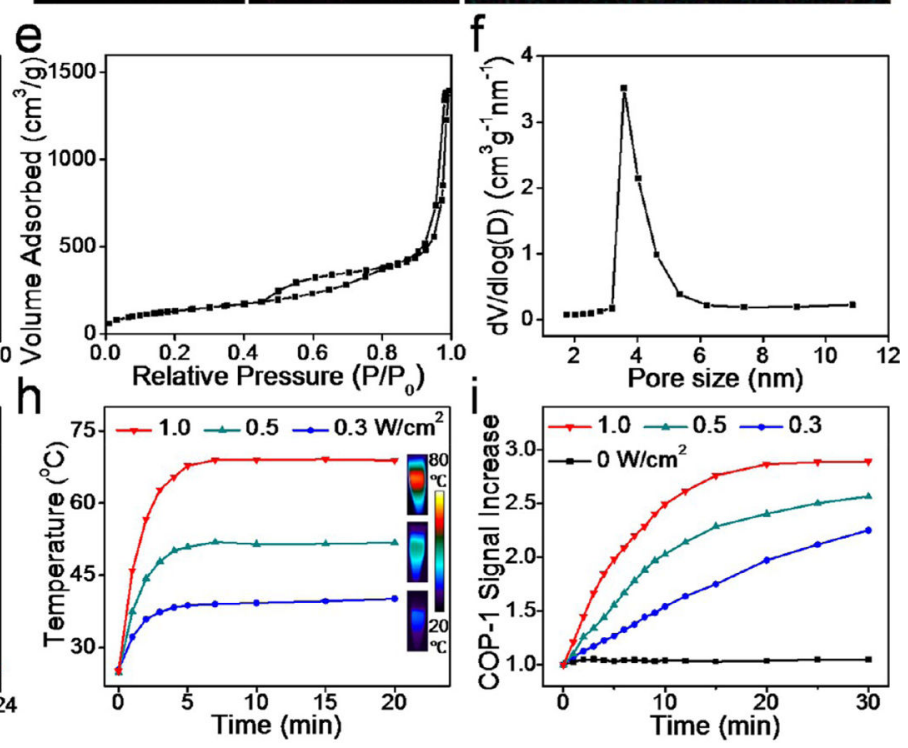

Figure 5.

Preparation and characterization of HMOPM-CO. (a) Schematic synthesis route. A typical $\mathrm{CO}$ releasing molecule, $\mathrm{Mn}_{2}(\mathrm{CO})_{10}$, was loaded into the $\mathrm{HMON}$ structures via hydrophobic -hydrophobic interaction. With GSH reduction, HMOPM-CO effectively converted NIR light energy into heat, which further triggered the decomposition of $\mathrm{Mn}_{2}(\mathrm{CO})_{10}$ for NIRcontrolled CO release. (b) TEM image. Scale bar, $50 \mathrm{~nm}$. (c) Elemental mapping. Scale bar, $50 \mathrm{~nm}$. (d) EDS spectrum confirmed the successful synthesis of HMOPM-CO by presenting both Mo and Mn. (e) $\mathrm{N}_{2}$ adsorption-desorption isotherm and (f) corresponding pore-size distribution. The surface area and pore size were determined to be $491.84 \mathrm{~m}^{2} / \mathrm{g}$ and around $3.7 \mathrm{~nm}$, respectively. (g) XPS spectrum of Mo 3d in HMOPM-CO. The Mo element was consistent with the valence of +6 , suggesting the $\mathrm{Mn}_{2}(\mathrm{CO})_{10}$ loading did not affect the subsequent POM surface modification. (h) Photothermal heating curves and images of HMOPM-CO solutions upon an $808 \mathrm{~nm}$ irradiation at different fluence rates. (i) The 
absorbance increases of COP-1 probe in response to $\mathrm{CO}$ generation in the irradiated HMOPM-CO aqueous solutions. The $\mathrm{CO}$ release amount was a function of the irradiation fluences and durations. 


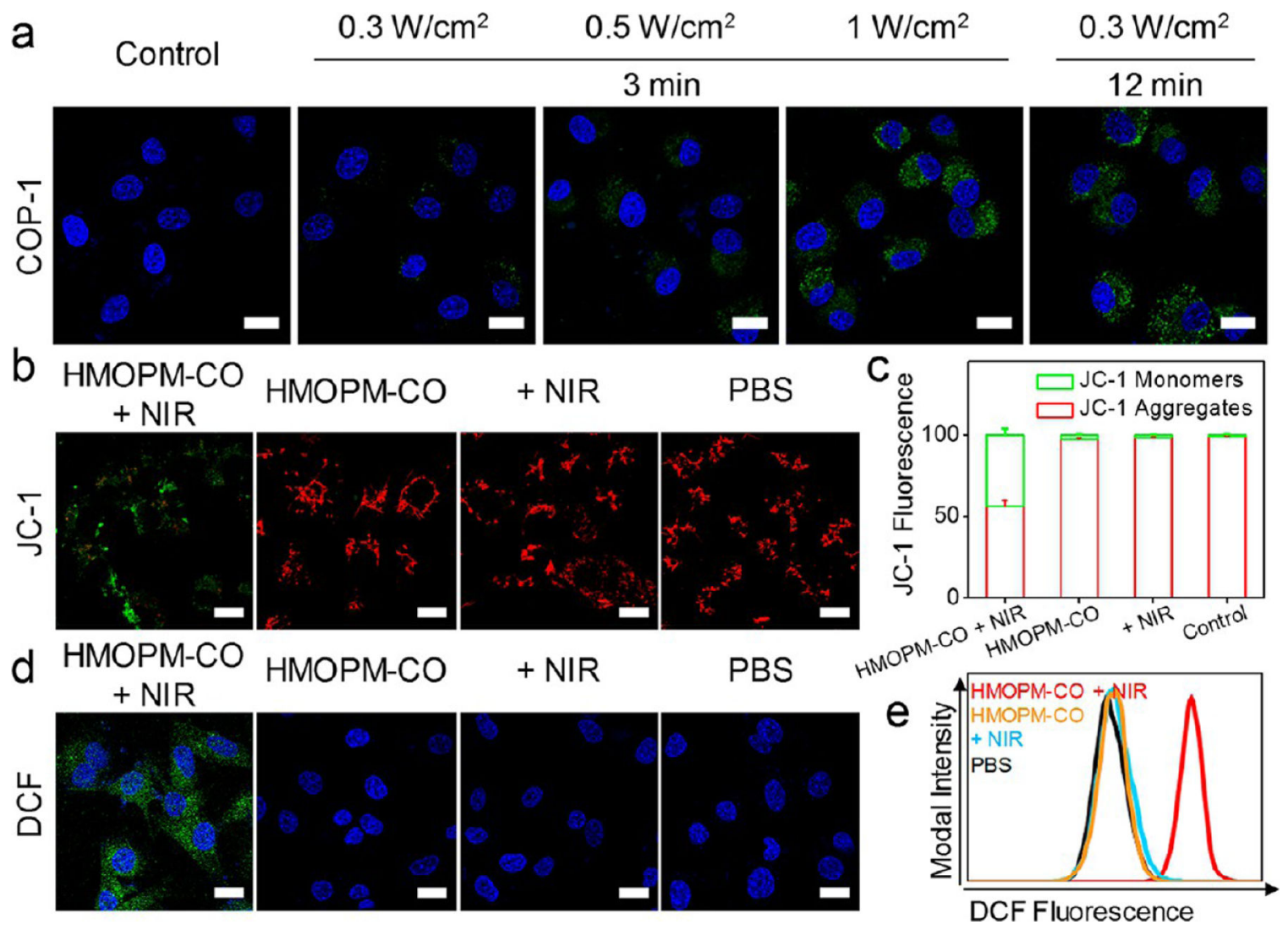

Figure 6.

In vitro NIR-controlled CO release. (a) Confocal fluorescence images of U87MG cells treated with HMOPM-CO (GSH preincubated) plus an $808 \mathrm{~nm}$ irradiation. COP-1 probe was used as a $\mathrm{CO}$ indicator. The $\mathrm{CO}$ release was dependent on both irradiation intensity and duration. A comparable level of positive COP-1 staining was observed in cells exposed to an irradiation of $0.3 \mathrm{~W} / \mathrm{cm}^{2}$ for $12 \mathrm{~min}$ or $1.0 \mathrm{~W} / \mathrm{cm}^{2}$ for $3 \mathrm{~min}$. Green, COP-1. Blue, DAPI. Scale bar, $20 \mu \mathrm{m}$. (b) JC-1 assay for illustrating the impact of the released $\mathrm{CO}$ on mitochondria. Cells were treated with HMOPM-CO (GSH preincubated) and then exposed to an $808 \mathrm{~nm}$ laser irradiation at $0.3 \mathrm{~W} / \mathrm{cm}^{2}$ for $12 \mathrm{~min}$. A significant decrease in red to green fluorescence ratio was observed, indicating mitochondrial depolarization. In contrast, all control groups showed overwhelming red fluorescence. Green, JC-1 monomers, low mitochondrial membrane potential of apoptotic or unhealthy cells. Red, JC-1 aggregates, high potential of healthy cells. Scale bar, $20 \mu \mathrm{m}$. (c) Flow cytometry quantitative analysis on mitochondrial membrane potential loss after different treatments. (d) $\mathrm{H}_{2}$ DCFDA assay for detecting intracellular ROS level. After the CO therapy, much stronger green fluorescence appeared in the cell, suggesting enhanced ROS generation. Green, DCF. Blue, DAPI. Scale bar, $20 \mu \mathrm{m}$. (e) Flow cytometry analysis of intracellular ROS generation. 

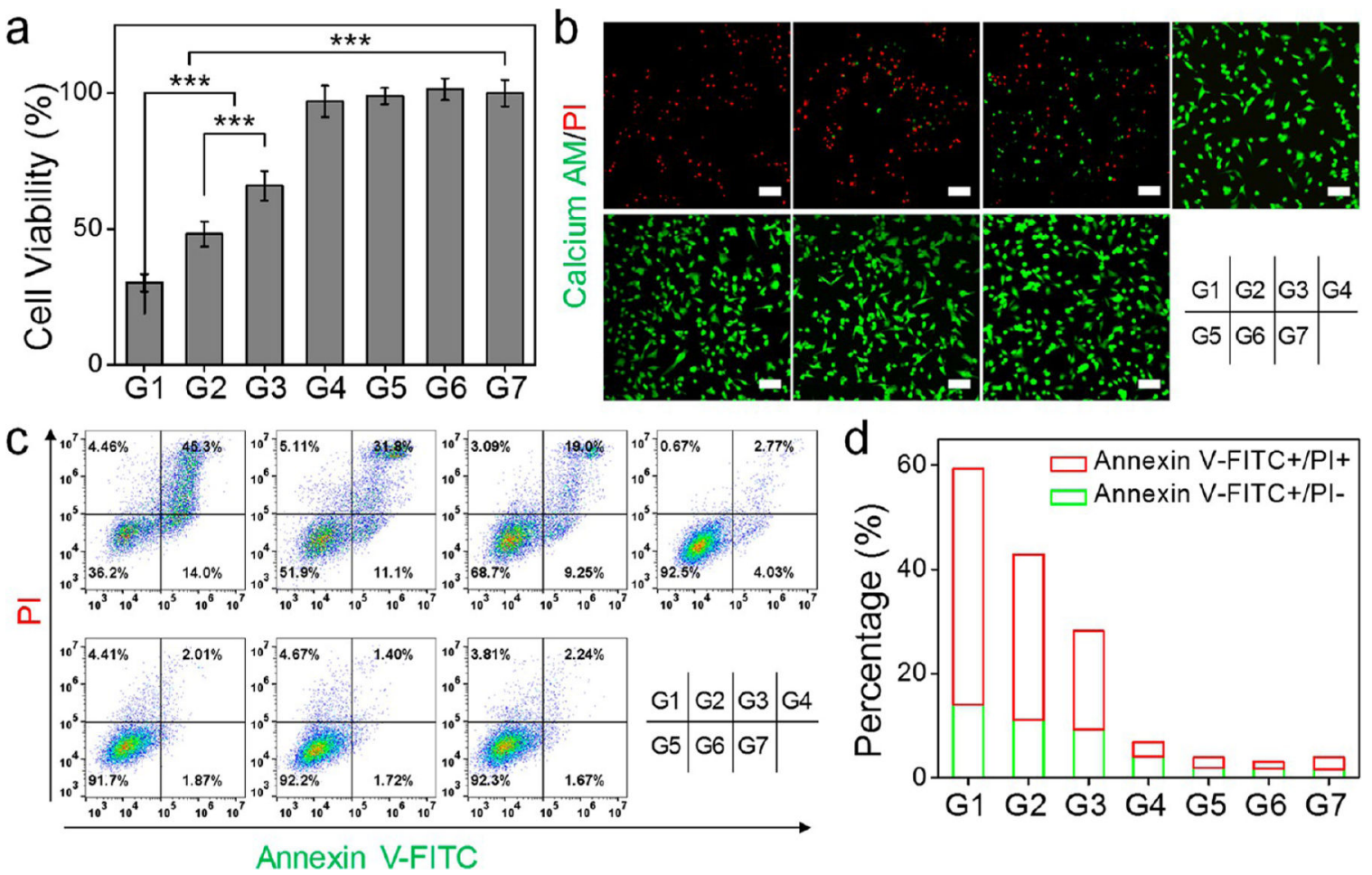

Figure 7.

In vitro evaluation of synergistic PTT-enhanced CO gas therapy on U87MG cells. Seven experimental groups were studied, with two $808 \mathrm{~nm}$ irradiation doses $\left(1.0 \mathrm{~W} / \mathrm{cm}^{2}\right.$ for $3 \mathrm{~min}$ or $0.3 \mathrm{~W} / \mathrm{cm}^{2}$ for $12 \mathrm{~min}$ ). G1, HMOPM-CO $+1.0 \mathrm{~W} / \mathrm{cm}^{2}$ (synergistic PTT/gas therapy group). G2, HMOPM + $1.0 \mathrm{~W} / \mathrm{cm}^{2}$ (PTT group). G3, HMOPM-CO $+0.3 \mathrm{~W} / \mathrm{cm}^{2}$ (gas therapy group). G4, HMOPM $+0.3 \mathrm{~W} / \mathrm{cm}^{2}$. G5, HMOPM-CO. G6, $+1.0 \mathrm{~W} / \mathrm{cm}^{2}$. G7, PBS. (a) MTT assays. (b) Live and dead assays. Green, Calcein AM, live cells. Red, PI, dead cells. Scale bar, $100 \mu \mathrm{m}$. (c) Flow cytometry analysis on apoptotic/necrotic cells via Annexin V-FITC/PI assays. (d) Quantitative analysis of corresponding cell apoptosis/ necrosis percentages based on (c). The synergistic PTT/gas therapy significantly improved the treatment efficacy. $* * * P<0.001$. 


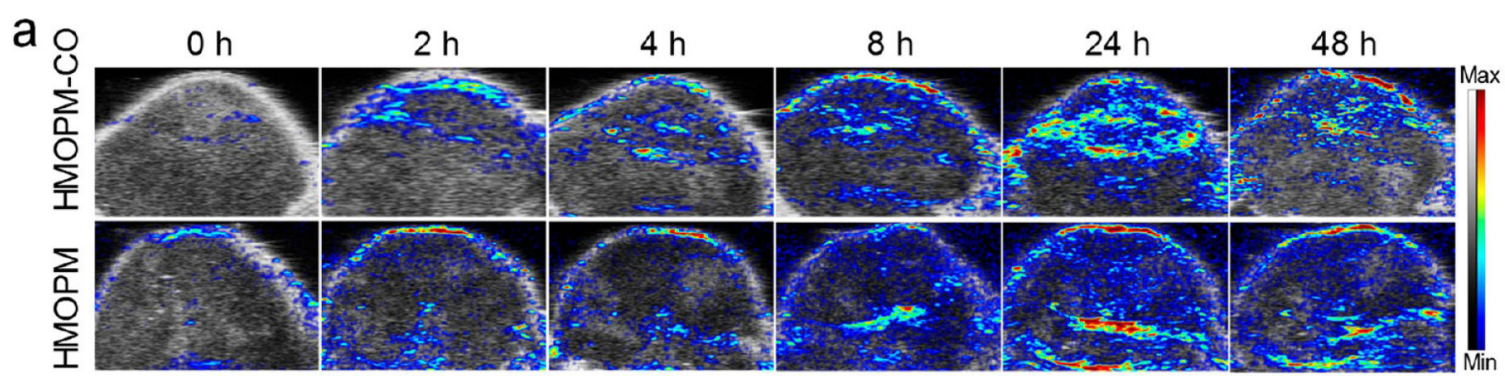

b $1 \mathrm{~W} / \mathrm{cm}^{2}, 5 \mathrm{~min}$
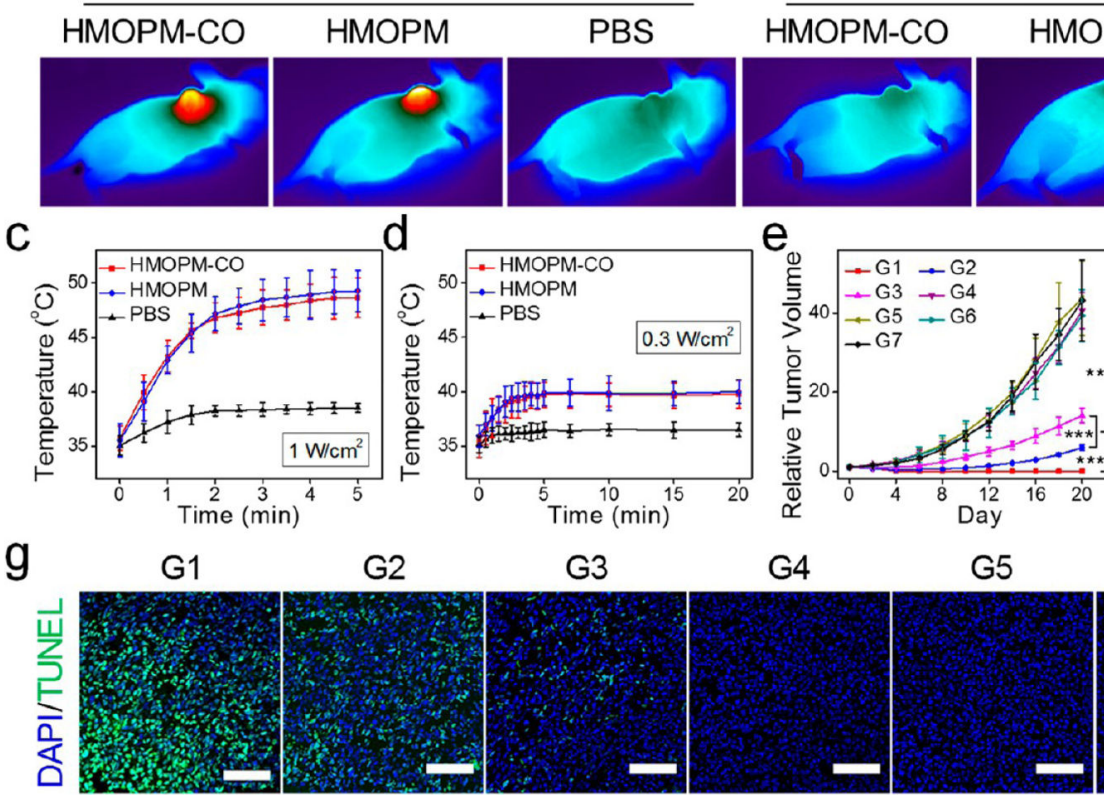

$0.3 \mathrm{~W} / \mathrm{cm}^{2}, 20 \mathrm{~min}$

Figure 8.

In vivo evaluation of the PA imaging and synergistic PTT-enhanced CO gas therapy with HMOPM-CO. (a) Representative PA images of U87MG tumors taken before and at different time points after intravenous injection of HMOPM-CO or HMOPM. The maximum PA intensity was observed at $24 \mathrm{~h}$ p.i. in both groups. (b) Thermographic images of mice at the end of an $808 \mathrm{~nm}$ irradiation at $24 \mathrm{~h}$ p.i. of HMOPM-CO, HMOPM, or PBS. (c, d)

Temperature rise curves $(\mathrm{n}=5$, mean \pm s.d.) at tumor regions during the irradiations at 1.0 $\mathrm{W} / \mathrm{cm}^{2}$ (c) or $0.3 \mathrm{~W} / \mathrm{cm}^{2}$ (d). A $1.0 \mathrm{~W} / \mathrm{cm}^{2}$ laser could significantly and rapidly heat the nanoparticle-treated tumors to around $50{ }^{\circ} \mathrm{C}$, whereas a $0.3 \mathrm{~W} / \mathrm{cm}^{2}$ laser only limitedly heated the tumors to a temperature below $40^{\circ} \mathrm{C}$. $(\mathrm{e}-\mathrm{g})$ Therapy studies with HMOPM-CO on U87MG tumor model $(n=5)$. Nanoparticles were intravenously administrated into the mice, and an $808 \mathrm{~nm}$ irradiation $\left(1.0 \mathrm{~W} / \mathrm{cm}^{2}\right.$ for $5 \mathrm{~min}$ or $0.3 \mathrm{~W} / \mathrm{cm}^{2}$ for $\left.20 \mathrm{~min}\right)$ was applied at $24 \mathrm{~h}$ p.i. Seven groups were studied. G1, HMOPM-CO $+1.0 \mathrm{~W} / \mathrm{cm}^{2}$ (synergistic PTT/gas therapy). G2, HMOPM $+1.0 \mathrm{~W} / \mathrm{cm}^{2}$ (PTT). G3, HMOPM-CO $+0.3 \mathrm{~W} / \mathrm{cm}^{2}$ (gas therapy). G4, HMOPM + $0.3 \mathrm{~W} / \mathrm{cm}^{2}$. G5, HMOPM-CO. G6, $+1.0 \mathrm{~W} / \mathrm{cm}^{2}$. G7, PBS. (e) Tumor growth curves and (f) tumor growth inhibition (TGI) rates of different treatments. Complete tumor eradication was achieved in the synergistic PTT-enhanced CO gas therapy group. The PTT or gas therapy alone also significantly suppressed the tumor growth with a TGI rate of $86.3 \%$ and $71.0 \%$, respectively, on day 20. (g) TUNEL assays on tumors acquired after $24 \mathrm{~h}$ 
of different treatments. A significantly higher level of apoptotic cells was found in the synergistic PTT/CO treatment group. Green, TUNEL. Blue, DAPI. Scale bar, $100 \mu \mathrm{m}$. *** $P$ $<0.001$. 